\title{
Fundamental Kinetics of Supercritical Coal Liquefaction: Effect of Catalysts and Hydrogen-Donor Solvents
}

\author{
Quarterly Report 11 \\ Reporting Period: April-June, 1997 \\ Principal Investigators: Ben J. McCoy, J.M. Smith \\ Students: Giridhar Madras, Yoichi Kodera \\ July 21, 1997 \\ GRANT NO. DOE DE-FG22-94PC94204 \\ Department of Chemical Engineering and Materials Science \\ University of California, Davis, CA 95616. (916) 752-1435
}

PERIOD OF GRANT AWARD: August, 1994 - July, 1998

\begin{abstract}
Disclaimer thereof.

\author{
Table of Contents \\ Disclaimer \\ Executive Summary \\ Abstract \\ Introduction \\ Experiments \\ Theoretical Model \\ Results and Discussion \\ Acknowledgement \\ References \\ Table 1 \\ Figure Captions
}

This report was prepared as an account of work sponsored by an agency of the United States Government. Neither the United States Government nor any agency thereof, nor any of their employees, makes any warranty, express or implied, or assumes any legal liability or responsibility for the accuracy, completeness, or usefulness of any information, apparatus, product, or process disclosed or represents that its use would not infringe privately owned rights. Reference herein to any specific commercial product, process or service by trade name, trademark, manufacturer, or otherwise does not necessarily constitute or imply its endorsement, recommendation, or favoring by the United States Government or any agency thereof. The views and opinions of authors expressed herein do not necessarily state or reflect those of the United States Government or any agency 


\section{Executive Summary}

This quarterly report shows our recent progress toward the overall objective to understand the supercritical fluid extraction of hydrocarbons from coal. Our strategy is to simulate coal as a high molecular-weight polymeric material by studying the degradation of polymers under various conditions. The hypothesis we are testing is that degradation of such macromolecules is applicable to the decomposition (depolymerization) of the coal network.

Polymer degradation and coal liquefaction are influenced strongly by the nature of the chemical compounds involved in the reaction. This motivated our investigation of the effect of mixtures on polymer degradation. In particular, we formulated a new theory and obtained new experimental data to show how adding a polymer can increase, decrease, or leave unchanged the degradation rate of the first polymer. In the current study, we examined the degradation of polymer mixtures by developing a detailed radical mechanism based on Rice-Herzfeld reactions. The interaction between different polymers occurs by radicals abstracting hydrogen atoms from either. Expressions for the degradation rates for each polymer were obtained by applying distribution kinetics to the MWD of the reacting radicals and polymers. As polymers degrade, predominantly by either chain-end scission or random-chain scission, three cases are possible for the interactive degradation of two polymers. Both polymers can degrade by chain-end scission, or by random-chain scission, or one polymer can degrade by random-chain scission and the other polymer by chain-end scission. In addition to developing the theory, we present new experimental data for the concentration effect of PAMS on polystyrene degradation in solution at $275^{\circ} \mathrm{C}$. 


\begin{abstract}
Most research on polymer degradation is for single polymers, even though the thermal decomposition of polymer mixtures is of interest both practically and theoretically. Polymer degradation rates depend on the mixture type, and adding a polymer can increase, decrease, or leave unchanged the degradation rate of the first polymer. We show how distribution-kinetics theory, based on molecular-weight distributions (MWDs), provides expressions for degradation rates of binary polymer mixtures. The approach accounts for initiation, termination, hydrogen abstraction, and radical chain scission in the governing equations for MWDs. Molecular-weight moments yield expressions for molar and mass concentrations and rate coefficients for combinations of random and chain-end scission. Experimental data show the concentration effect of poly( $\alpha$-methyl styrene) (PAMS) on the degradation of polystyrene dissolved in mineral oil at $275^{\circ} \mathrm{C}$ in a batch reactor. Samples analyzed by gel permeation chromatography yielded the time evolution of the MWD. The results indicated that, owing to the interaction of mixed radicals with polymer by hydrogen abstraction, polystyrene degradation rate decreases with increasing PAMS concentration.
\end{abstract}




\section{Introduction}

Disposal of plastic wastes has become a worldwide problem and the cost of conventional disposal is likely to increase ( $\mathrm{Ng}$ et al., 1995). Recycling of polymers may be a viable alternative and thermochemical recycling of polymers has received growing attention in recent years (Miller, 1994). Understanding the thermal decomposition of polymers is also an important issue in polymer science and engineering (Clough et al., 1996). Most research has focused on the mechanism of degradation of single polymers. However, waste streams usually contain mixtures of polymers and it is costly to separate them prior to degradation (McCaffrey et al., 1996). Thus it is important to study the degradation of polymer mixtures. The polymer degradation rate can be modified by the addition of conventional free-radical initiators, oxidizers or hydrogen donors but it might be easier to alter the degradation rate by blending two polymers (Gardner et al., 1993).

Degradation studies by pyrolysis of polymer mixtures have reported varied results. For example, some reports indicated a significant interaction between polystyrene and polyethylene (Koo and Kim, 1993; Koo et al., 1991; McCaffrey et al., 1996), while others observed no interaction between these polymers (Roy et al., 1978; Wu et al., 1993). The pyrolytic polystyrene degradation rate was significantly enhanced in the presence of poly(methyl acrylate) and poly(butyl acrylate) at $430{ }^{\circ} \mathrm{C}$ (Gardner et al., 1993). Richards and Salter (1964), on the other hand, observed that the rate of polystyrene degradation decreased with increasing molecular weight (MW) of added PAMS. These reports suggest the need for an analysis of the underlying reaction mechanisms.

Degradation of polymers in solution has been proposed to ameliorate problems encountered in commercial applications (Sato et al., 1990). The degradation of polystyrene (Murakata et al., 1993; Madras et al., 1996c), poly(styrene-allyl alcohol) (Wang et al., 1995), poly(methyl methacrylate) (Madras et al., 1996a), PAMS (Madras et al., 1996b) in solution have been investigated. No studies on the degradation of polymer mixtures in solution, however, have been reported. 
In the current study, we examined the degradation of polymer mixtures by developing a detailed radical mechanism based on Rice-Herzfeld reactions (Nigam et al., 1994). The interaction between different polymers occurs by radicals abstracting hydrogen atoms from either polymer (McCaffrey et al., 1996). Expressions for the degradation rates for each polymer were obtained by applying distribution kinetics to the MWD of the reacting radicals and polymers. As polymers degrade, predominantly by either chain-end scission or random-chain scission, three cases are possible for the interactive degradation of two polymers. Both polymers can degrade by chain-end scission, or by random-chain scission, or one polymer can degrade by random-chain scission and the other polymer by chain-end scission. In addition to developing a theory, we present new experimental data for the concentration effect of PAMS on polystyrene degradation in solution at $275^{\circ} \mathrm{C}$.

\section{Experiments}

Analysis of MWDs. The HPLC (Hewlett-Packard 1050) system consists of a $100 \mu \mathrm{L}$ sample loop, a gradient pump, and an on-line variable wavelength ultraviolet (UV) detector. Three PLgel columns (Polymer Lab Inc.) (300 mm x $7.5 \mathrm{~mm}$ ) packed with

cross-linked poly(styrene-divinyl benzene) with pore sizes of 100,500 , and $10^{4} \AA$ are used in series. Tetrahydrofuran (HPLC grade, Fisher Chemicals) was pumped at a constant flow rate of $1.00 \mathrm{~mL} / \mathrm{min}$. Narrow MW polystyrene standards of MW 162 to 0.93 million (Polymer Lab and Aldrich Chemicals) were used to obtain the calibration curve of retention time versus MW (Figure 1), which was stable during the period of the experiments. The calibration curve, modeled as a second-order polynomial, indicates a higher accuracy in the measurement of lower MW polymers.

Polystyrene Degradation. The thermal decomposition of polystyrene in mineral oil was conducted in a $100 \mathrm{~mL}$ flask equipped with a reflux condenser to ensure the condensation and retention of volatiles. To observe a significant effect of PAMS on the conversion, polystyrene of high MW was chosen. $60 \mathrm{~mL}$ of mineral oil (Fisher Chemicals) was heated 
to $275^{\circ} \mathrm{C}$, and various amounts $(0-0.60 \mathrm{~g})$ of monodisperse PAMS (MW $=11,000$, Scientific Polymer Products), and $0.12 \mathrm{~g}$ of monodisperse polystyrene (MW = 330,000, Aldrich Chemicals) were added. The temperature of the solution was measured with a Type $\mathrm{K}$ thermocouple (Fisher Chemicals) and controlled within $\pm 1^{\circ} \mathrm{C}$ by a Omega CN2042 temperature controller. Samples of $1.0 \mathrm{~mL}$ were taken at 15 minute intervals and dissolved in $1.0 \mathrm{~mL}$ of tetrahydrofuran, THF (HPLC grade, Fisher Chemicals). The chromatograph obtained by injecting $100 \mu \mathrm{L}$ of this solution into the HPLC-GPC system was converted to MWD. The peaks of the reacted polystyrene, PAMS, and the oligomers were distinct, so that moments could be calculated by numerical integration. Because the solvent mineral oil is UV invisible, its MWD was determined with a refractive index (RI) detector. No change in the MWD of mineral oil (Figure 2) was observed when the oil was heated for 3 hours at $275^{\circ} \mathrm{C}$ without polystyrene.

\section{Theoretical Model}

According to the Rice-Herzfeld mechanism, polymers can transform without change in MW by hydrogen abstraction. Their radicals can also undergo chain scission to form lower MW products, or undergo addition reactions yielding higher MW products. Chain scission can occur either at the chain end yielding a specific product, or at a random position along the chain yielding a range of lower MW products.

Distribution kinetics is a straightforward and effective technique to represent macromolecular reactions. Continuous-distribution mass balances are written for the various steps involved in the radical mechanism (Table 1). The rate coefficients are here assumed to be independent of MW, a reasonable assumption at low conversions (Madras et al., 1996b). The integro-differential equations obtained from the mass balances can be solved for MW moments. These moment equations are usually coupled ordinary differential equations that, in general, can be solved numerically. 
In the present treatment, two common assumptions simplify the governing equations. The long-chain approximation (LCA) (Nigam et al., 1994; Gavalas, 1966) postulates that the initiation and termination rates are negligible because such events are infrequent compared to hydrogen abstraction and propagation-depropagation chain reactions. The quasi-stationary state approximation (QSSA) applies when radical concentrations are extremely small and their rates of change are negligible. The proposed scheme, based on the Rice-Herzfeld (Nigam et al., 1994) concept of chain reactions, includes the elementary steps of initiation, depropagation, hydrogen abstraction, and termination.

Case I. One polymer undergoes random-chain scission, the other polymer undergoes chain-end scission and both interact with each other.

The degradation rate of polymer A undergoing random-chain scission is influenced by polymer B undergoing chain-end scission. We represent the reacting polymer A and its radicals as $P_{A}(x)$ and $R^{\circ}(x)$ and their MWDs as $p_{A}(x, t)$ and $r(x, t)$, respectively, where $x$ represents the continuous variable, MW. As the polymer reactants and random scission products are not distinguished in the distribution kinetics model, a single MWD, $\mathrm{p}_{\mathrm{A}}(\mathrm{x}, \mathrm{t})$, represents the polymer in the mixture at any time, $t$. The initiation-termination reactions are represented as

$$
\begin{gathered}
\mathrm{k}_{\mathrm{f}} \\
\mathrm{P}_{\mathrm{A}}(\mathrm{x}) \Leftrightarrow \mathrm{R}^{\bullet}\left(\mathrm{x}^{\prime}\right)+\mathrm{R}^{\bullet}\left(\mathrm{x}-\mathrm{x}^{\prime}\right) \\
\mathrm{k}_{\mathrm{t}}
\end{gathered}
$$

where $\Leftrightarrow$ represents a reversible reaction. The reversible hydrogen abstraction process is

$$
\begin{aligned}
& \mathrm{k}_{\mathrm{h}} \\
& \mathrm{P}_{\mathrm{A}}(\mathrm{x}) \Leftrightarrow \mathrm{R}^{\bullet}(\mathrm{x}) \\
& \mathrm{k}_{\mathrm{H}}
\end{aligned}
$$

The depropagation chain reaction is

$$
\begin{gathered}
\mathrm{k}_{\mathrm{b}} \\
\mathrm{R}^{\bullet}(\mathrm{x}) \rightarrow \mathrm{P}_{\mathrm{A}}\left(\mathrm{x}^{\prime}\right)+\mathrm{R}^{\bullet}\left(\mathrm{x}-\mathrm{x}^{\prime}\right)
\end{gathered}
$$


The polymer B, the chain-end radical, the specific radical, and the specific product are represented as $\mathrm{P}_{\mathrm{B}}(\mathrm{x}), \mathrm{R}_{\mathrm{e}}{ }^{\cdot}(\mathrm{x}), \mathrm{R}_{\mathrm{S}}{ }^{\bullet}(\mathrm{x}), \mathrm{Q}_{\mathrm{S}}\left(\mathrm{x}_{\mathrm{S}}\right)$, respectively, and their corresponding MWDs as $\mathrm{p}_{\mathrm{B}}(\mathrm{x}, \mathrm{t}), \mathrm{r}_{\mathrm{e}}(\mathrm{x}, \mathrm{t}), \mathrm{r}_{\mathrm{s}}(\mathrm{x}, \mathrm{t})$ and $\mathrm{q}_{\mathrm{s}}\left(\mathrm{x}_{\mathrm{s}}, \mathrm{t}\right)$. The formation of chain-end radicals by a reversible random-scission initiation-termination reaction is

$$
\mathrm{P}_{\mathrm{B}}(\mathrm{x}) \underset{\mathrm{k}_{\mathrm{ts}}}{\Leftrightarrow} \mathrm{R}_{\mathrm{e}} \cdot(\mathrm{x})+\mathrm{R}_{\mathrm{e}} \cdot\left(\mathrm{x}-\mathrm{x}^{\prime}\right)
$$

Hydrogen abstraction by the chain-end radical is considered reversible, $\mathrm{k}_{\mathrm{he}}$

$$
\mathrm{P}_{\mathrm{B}}(\mathrm{x}) \underset{\mathrm{k}_{\mathrm{He}}}{\Leftrightarrow} \mathrm{R}_{\mathrm{e}}{ }^{\cdot(\mathrm{x})}
$$

The chain-end radical can undergo radical isomerization via a cyclic transition state to form a specific radical,

$$
R_{e^{\cdot}} \stackrel{k_{i h}}{\Leftrightarrow} \underset{k_{i H}}{\Leftrightarrow} R_{s^{\cdot}}(x)
$$

The depropagation reaction yields the specific product and a chain-end radical from a specific radical,

$$
\mathrm{R}_{\mathrm{S}} \mathrm{k}_{\mathrm{bs}} \mathrm{x}^{\rightarrow} \mathrm{Q}_{\mathrm{s}}\left(\mathrm{x}_{\mathrm{S}}\right)+\mathrm{R}_{\mathrm{e}} \cdot\left(\mathrm{x}-\mathrm{x}_{\mathrm{S}}\right)
$$

where $\mathrm{x}_{\mathrm{S}}$ is the MW of the specific product.

The interaction of the two degrading polymers is through hydrogen abstraction (McCaffrey et al., 1996) represented as a reversible disproportionation reaction. The end radical of polymer B combines with polymer A to form an intermediate radical complex that undergoes transformation to polymer B and a radical of polymer A,

$$
\begin{aligned}
& \mathrm{k}_{\mathrm{d}} \quad \mathrm{k}_{\mathrm{D}} \\
& \mathrm{R}_{\mathrm{e}}{ }^{\cdot}(\mathrm{x})+\mathrm{P}_{\mathrm{A}}\left(\mathrm{x}^{\prime}\right) \Leftrightarrow \mathrm{R}_{\mathrm{i}}{ }^{\cdot}\left(\mathrm{x}+\mathrm{x}^{\prime}\right) \Leftrightarrow \mathrm{P}_{\mathrm{B}}(\mathrm{x})+\mathrm{R}^{\bullet}\left(\mathrm{x}^{\prime}\right) \\
& \mathrm{k}_{\mathrm{D}} \quad \mathrm{k}_{\mathrm{d}}
\end{aligned}
$$


Including the intermediate complex, $\mathrm{R}_{\mathrm{i}}{ }^{\circ}$, facilitates the formulation of the population balance equations for the reversible disproportionation. If $\mathrm{R}_{\mathrm{i}}{ }^{\bullet}$ is ignored in equation 1.8 , the forward and reverse rate coefficients would be $\mathrm{k}_{\mathrm{d}}$ and $\mathrm{k}_{\mathrm{D}}$, respectively.

With the aid of Table 1, the rate expressions and moments can be formulated. Based on LCA, $\mathrm{k}_{\mathrm{f}}, \mathrm{k}_{\mathrm{fs}}, \mathrm{k}_{\mathrm{t}}$ and $\mathrm{k}_{\mathrm{ts}}$ are set to zero. The population balance equations for gain (+) and loss (-) terms are

$$
\begin{aligned}
& \partial \mathrm{p}_{\mathrm{A}} / \partial \mathrm{t}=-\mathrm{k}_{\mathrm{h}} \mathrm{p}_{\mathrm{A}}(\mathrm{x})+\mathrm{k}_{\mathrm{H}} \mathrm{r}(\mathrm{x})+\mathrm{k}_{\mathrm{b}} \int_{\mathrm{x}}^{\infty} \mathrm{r}\left(\mathrm{x}^{\prime}\right) \Omega\left(\mathrm{x}, \mathrm{x}^{\prime}\right) \mathrm{dx^{ \prime }}-\mathrm{k}_{\mathrm{d}} \mathrm{p}_{\mathrm{A}} \mathrm{r}_{\mathrm{e}}^{(0)}(\mathrm{x}) \\
& +\mathrm{k}_{\mathrm{D}} \int_{\mathrm{x}}^{\infty} \mathrm{r}_{\mathrm{i}}\left(\mathrm{x}^{\prime}\right) \Omega\left(\mathrm{x}, \mathrm{x}^{\prime}\right) \mathrm{d} \mathrm{x}^{\prime} \\
& \partial \mathrm{p}_{\mathrm{B}} / \partial \mathrm{t}=-\mathrm{k}_{\mathrm{he}} \mathrm{p}_{\mathrm{B}}(\mathrm{x})+\mathrm{k}_{\mathrm{He}} \mathrm{r}_{\mathrm{e}}(\mathrm{x})-\mathrm{k}_{\mathrm{d}} \mathrm{p}_{\mathrm{B}} \mathrm{r}^{(0)}(\mathrm{x})+\mathrm{k}_{\mathrm{D}} \int_{\mathrm{x}}^{\infty} \mathrm{r}_{\mathrm{i}}\left(\mathrm{x}^{\prime}\right) \Omega\left(\mathrm{x}, \mathrm{x}^{\prime}\right) \mathrm{d} \mathrm{x}^{\prime} \\
& \partial \mathrm{q}_{\mathrm{s}} / \partial \mathrm{t}=\mathrm{k}_{\mathrm{bs}} \int_{\mathrm{x}}^{\infty} \mathrm{r}_{\mathrm{s}}\left(\mathrm{x}^{\prime}\right) \delta\left(\mathrm{x}^{\prime}-\mathrm{x}_{\mathrm{s}}\right) \mathrm{dx^{ \prime }} \\
& \partial \mathrm{r}_{\mathrm{s}} / \partial \mathrm{t}=\mathrm{k}_{\mathrm{ih}} \mathrm{r}_{\mathrm{e}}(\mathrm{x})-\mathrm{k}_{\mathrm{iH}} \mathrm{r}_{\mathrm{s}}(\mathrm{x})-\mathrm{k}_{\mathrm{bs}} \mathrm{r}_{\mathrm{s}}(\mathrm{x}) \\
& \partial \mathrm{r}_{\mathrm{e}} / \partial \mathrm{t}=\mathrm{k}_{\mathrm{he}} \mathrm{p}_{\mathrm{B}}(\mathrm{x})-\mathrm{k}_{\mathrm{He}} \mathrm{r}_{\mathrm{e}}(\mathrm{x})-\mathrm{k}_{\mathrm{ih}} \mathrm{r}_{\mathrm{e}}(\mathrm{x})+\mathrm{k}_{\mathrm{iH}} \mathrm{r}_{\mathrm{s}}(\mathrm{x}) \\
& +\mathrm{k}_{\mathrm{bs}} \int_{\mathrm{x}}^{\infty} \mathrm{r}_{\mathrm{s}}\left(\mathrm{x}^{\prime}\right) \delta\left[\mathrm{x}-\left(\mathrm{x}^{\prime}-\mathrm{x}_{\mathrm{s}}\right)\right] \mathrm{dx^{ \prime }}-\mathrm{k}_{\mathrm{d}} \mathrm{p}_{\mathrm{A}}{ }^{(0)} \mathrm{r}_{\mathrm{e}}(\mathrm{x}) \\
& +\mathrm{k}_{\mathrm{D}} \int_{\mathrm{x}}^{\infty} \mathrm{r}_{\mathrm{i}}\left(\mathrm{x}^{\prime}\right) \Omega\left(\mathrm{x}, \mathrm{x}^{\prime}\right) \mathrm{d} \mathrm{x}^{\prime} \\
& \partial \mathrm{r} / \partial \mathrm{t}=\mathrm{k}_{\mathrm{h}} \mathrm{p}_{\mathrm{A}}(\mathrm{x})-\mathrm{k}_{\mathrm{H}} \mathrm{r}(\mathrm{x})-\mathrm{k}_{\mathrm{b}} \mathrm{r}(\mathrm{x})+\mathrm{k}_{\mathrm{b}} \int_{\mathrm{x}}^{\infty} \mathrm{r}\left(\mathrm{x}^{\prime}\right) \Omega\left(\mathrm{x}, \mathrm{x}^{\prime}\right) \mathrm{dx}^{\prime}-\mathrm{k}_{\mathrm{d}} \mathrm{p}_{\mathrm{B}}{ }^{(0)} \mathrm{r}(\mathrm{x}) \\
& +\mathrm{k}_{\mathrm{D}} \int_{\mathrm{x}}^{\infty} \mathrm{r}_{\mathrm{i}}\left(\mathrm{x}^{\prime}\right) \Omega\left(\mathrm{x}, \mathrm{x}^{\prime}\right) \mathrm{dx} \mathrm{x}^{\prime} \\
& \partial \mathrm{r}_{\mathrm{i}} / \partial \mathrm{t}=\mathrm{k}_{\mathrm{d}} \int_{0}^{\mathrm{x}} \mathrm{p}_{\mathrm{A}}\left(\mathrm{x}^{\prime}\right) \mathrm{r}_{\mathrm{e}}\left(\mathrm{x}-\mathrm{x}^{\prime}\right) \mathrm{d} \mathrm{x}^{\prime}+\mathrm{k}_{\mathrm{d}} \int_{0}^{\mathrm{x}} \mathrm{p}_{\mathrm{B}}\left(\mathrm{x}^{\prime}\right) \mathrm{r}\left(\mathrm{x}-\mathrm{x}^{\prime}\right) \mathrm{d} \mathrm{x}^{\prime}-2 \mathrm{k}_{\mathrm{D}} \mathrm{r}_{\mathrm{i}}(\mathrm{x})
\end{aligned}
$$

The moments for the polymer, radicals and the monomer, $\mathrm{p}^{(\mathrm{n})}, \mathrm{r}^{(\mathrm{n})}, \mathrm{q}^{(\mathrm{n})}$, respectively, are defined as

$$
\begin{aligned}
& \mathrm{p}^{(\mathrm{n})}(\mathrm{t})=\int_{0}^{\infty} \mathrm{x}^{\mathrm{n}} \mathrm{p}(\mathrm{x}, \mathrm{t}) \mathrm{dx} \\
& \mathrm{r}^{(\mathrm{n})}(\mathrm{t})=\int_{0}^{\infty} \mathrm{x}^{\mathrm{n}} \mathrm{r}(\mathrm{x}, \mathrm{t}) d x \\
& \mathrm{q}^{(\mathrm{n})}(\mathrm{t})=\int_{0}^{\infty} \mathrm{x}^{\mathrm{n}} \mathrm{q}(\mathrm{x}, \mathrm{t}) \mathrm{dx}
\end{aligned}
$$

Applying the moment operation, $\int_{0}^{\infty}[] \mathrm{x}^{\mathrm{n}} \mathrm{dx}$, to each balance equation yields

$$
\begin{aligned}
& \mathrm{dp}_{\mathrm{A}}{ }^{(\mathrm{n})} / \mathrm{dt}=-\mathrm{k}_{\mathrm{h}} \mathrm{p}_{\mathrm{A}}{ }^{(\mathrm{n})}+\mathrm{k}_{\mathrm{H}} \mathrm{r}^{(\mathrm{n})}+\mathrm{k}_{\mathrm{b}} \mathrm{Z}_{\mathrm{n} 0} \mathrm{r}^{(\mathrm{n})}-\mathrm{k}_{\mathrm{d}} \mathrm{p}_{\mathrm{A}}{ }^{(\mathrm{n})} \mathrm{r}_{\mathrm{e}}{ }^{(0)} \\
& +\mathrm{k}_{\mathrm{D}} \mathrm{Z}_{\mathrm{n} 0} \mathrm{r}_{\mathrm{i}}^{(\mathrm{n})} \\
& \mathrm{dp}_{\mathrm{B}}{ }^{(\mathrm{n})} / \mathrm{dt}=-\mathrm{k}_{\mathrm{he}} \mathrm{p}_{\mathrm{B}}{ }^{(\mathrm{n})}+\mathrm{k}_{\mathrm{He}} \mathrm{r}_{\mathrm{e}}^{(\mathrm{n})}-\mathrm{k}_{\mathrm{d}} \mathrm{p}_{\mathrm{B}}{ }^{(\mathrm{n}) \mathrm{r}^{(0)}}+\mathrm{k}_{\mathrm{D}} \mathrm{Z}_{\mathrm{n} 0} \mathrm{r}_{\mathrm{i}}{ }^{(\mathrm{n})}
\end{aligned}
$$




$$
\begin{aligned}
& \mathrm{dq}_{\mathrm{s}}{ }^{(\mathrm{n}) / \mathrm{dt}}=\mathrm{k}_{\mathrm{bs}} \mathrm{x}_{\mathrm{s}}{ }^{\mathrm{n}} \mathrm{r}_{\mathrm{s}}{ }^{(0)} \\
& \mathrm{dr}_{\mathrm{S}}^{(\mathrm{n})} / \mathrm{dt}=\mathrm{k}_{\mathrm{ih}} \mathrm{r}_{\mathrm{e}}^{(\mathrm{n})}-\mathrm{k}_{\mathrm{iH}} \mathrm{r}_{\mathrm{s}}^{(\mathrm{n})}-\mathrm{k}_{\mathrm{bs}} \mathrm{r}_{\mathrm{s}}^{(\mathrm{n})} \\
& \mathrm{dr}_{\mathrm{e}}^{(\mathrm{n})} / \mathrm{dt}=\mathrm{k}_{\mathrm{he}} \mathrm{p}_{\mathrm{B}}^{(\mathrm{n})}-\mathrm{k}_{\mathrm{He}} \mathrm{r}_{\mathrm{e}}^{(\mathrm{n})}-\mathrm{k}_{\mathrm{ih}} \mathrm{r}_{\mathrm{e}}^{(\mathrm{n})}+\mathrm{k}_{\mathrm{iH}} \mathrm{r}_{\mathrm{s}}^{(\mathrm{n})} \\
& +k_{b s} \sum_{j=0}^{n}\left({ }_{j}^{n}\right)\left(x_{s}\right)^{j}(-1)^{j} r_{s}{ }^{(n-j)}-k_{d} p_{A}{ }^{(0)} r_{e}{ }^{(n)}+k_{D} Z_{n 0} r_{i}{ }^{(n)} \\
& \mathrm{dr}^{(\mathrm{n})} / \mathrm{dt}=\mathrm{k}_{\mathrm{h}} \mathrm{p}_{\mathrm{A}}{ }^{(\mathrm{n})}-\mathrm{k}_{\mathrm{H}} \mathrm{r}^{(\mathrm{n})}-\mathrm{k}_{\mathrm{b}} \mathrm{r}^{(\mathrm{n})}+\mathrm{k}_{\mathrm{b}} \mathrm{Z}_{\mathrm{n} 0} \mathrm{r}^{(\mathrm{n})}-\mathrm{k}_{\mathrm{d}} \mathrm{p}_{\mathrm{B}}{ }^{(0)} \mathrm{r}^{(\mathrm{n})} \\
& +\mathrm{k}_{\mathrm{D}} \mathrm{Z}_{\mathrm{n} 0} \mathrm{r}_{\mathrm{i}}^{(\mathrm{n})} \\
& d_{i}{ }^{(n)} / d t=k_{d} \sum_{j=0}^{n}\left({ }_{j}^{n}\right) r_{e}^{(n-j)} p_{A}^{(j)}+k_{d} \sum_{j=0}^{n}\left({ }_{j}^{n}\right) r^{(n-j)} p_{B}^{(j)}-2 k_{D} r_{i}^{(n)}
\end{aligned}
$$

where $Z_{n 0}=1 /(n+1)$. The initial conditions for moments are

$$
\mathrm{p}_{\mathrm{B}}{ }^{(\mathrm{n})}(\mathrm{t}=0)=\mathrm{p}_{\mathrm{B} 0}{ }^{(\mathrm{n})}, \mathrm{p}_{\mathrm{A}}{ }^{(\mathrm{n})}(\mathrm{t}=0)=\mathrm{p}_{\mathrm{A} 0}{ }^{(\mathrm{n})} \text { and } \mathrm{r}^{(\mathrm{n})}(\mathrm{t}=0)=0
$$

When QSSA holds,

$$
\mathrm{dr}^{(\mathrm{n})} / \mathrm{dt}=\mathrm{dr}_{\mathrm{s}}^{(\mathrm{n})} / \mathrm{dt}=\mathrm{dr}_{\mathrm{e}}^{(\mathrm{n})} / \mathrm{dt}=\mathrm{dr}_{\mathrm{i}}^{(\mathrm{n})} / \mathrm{dt}=0
$$

Expressions for the radical concentrations can be obtained by equating the expressions of the zeroth moments $(n=0$ in equations $1.22-1.25)$ to zero. Equation 1.22 yields

$$
\mathrm{r}_{\mathrm{s}}^{(0)}=\mathrm{r}_{\mathrm{e}}^{(0)} \mathrm{k}_{\mathrm{ih}} /\left(\mathrm{k}_{\mathrm{bs}}+\mathrm{k}_{\mathrm{iH}}\right)
$$

Equation 1.25 yields

$$
\mathrm{r}_{\mathrm{i}}^{(0)}=\mathrm{k}_{\mathrm{d}}\left(\mathrm{r}_{\mathrm{e}}^{(0)} \mathrm{p}_{\mathrm{A}}^{(0)}+\mathrm{r}^{(0)} \mathrm{p}_{\mathrm{B}}^{(0)}\right) /\left(2 \mathrm{k}_{\mathrm{D}}\right)
$$

Equations 1.23 and 1.24 are solved simultaneously to obtain,

$$
\begin{array}{r}
\mathrm{r}^{(0)}=\mathrm{p}_{\mathrm{A}}^{(0)}\left(2 \mathrm{k}_{\mathrm{h}}+\mathrm{k}_{\mathrm{d}} \mathrm{r}_{\mathrm{e}}^{(0)}\right) /\left(2 \mathrm{k}_{\mathrm{H}}+\mathrm{k}_{\mathrm{d}} \mathrm{p}_{\mathrm{B}}^{(0)}\right) \\
\mathrm{r}_{\mathrm{e}}^{(0)}=\mathrm{p}_{\mathrm{B}}^{(0)}\left(2 \mathrm{k}_{\mathrm{he}} \mathrm{k}_{\mathrm{H}}+\mathrm{k}_{\mathrm{d}}\left(\mathrm{k}_{\mathrm{he}}+\mathrm{k}_{\mathrm{h}}\right) \mathrm{p}_{\mathrm{A}}{ }^{(0)}\right) / \\
\quad\left(2 \mathrm{k}_{\mathrm{He}} \mathrm{k}_{\mathrm{H}}+\mathrm{k}_{\mathrm{d}}\left(\mathrm{k}_{\mathrm{He}} \mathrm{p}_{\mathrm{B}}^{(0)}+\mathrm{k}_{\mathrm{H}} \mathrm{p}_{\mathrm{A}}{ }^{(0)}\right)\right)
\end{array}
$$

Because PAMS degrades only by chain-end scission, the zeroth moment (molar concentration) of the polymer is a constant (Madras et al., 1996b),

$$
\mathrm{dp}_{\mathrm{B}}{ }^{(0)} / \mathrm{dt}=0
$$

and therefore,

$$
\mathrm{p}_{\mathrm{B}}{ }^{(0)}=\mathrm{p}_{\mathrm{B} 0}{ }^{(0)}
$$


Substituting equations $1.28-1.31$ in equation 1.19 yields

$$
\mathrm{dp}_{\mathrm{A}}{ }^{(0)} / \mathrm{dt}=\mathrm{k}_{\mathrm{b}} \mathrm{r}^{(0)}
$$

where $\mathrm{r}^{(0)}$ and $\mathrm{r}_{\mathrm{e}}{ }^{(0)}$ are given by equations $1.30,1.31$ with $\mathrm{p}_{\mathrm{B}}{ }^{(0)}=\mathrm{p}_{\mathrm{B} 0}{ }^{(0)}$. The differential equation 1.34 can be solved, as discussed in the results and discussion section, when reasonable assumptions are made for the primary reaction rate coefficients.

The summation of first moments $(\mathrm{n}=1)$ for polymer and radicals (total mass concentration) must be constant when there are no losses from the reactor. For $\mathrm{n}=1$, equations $1.19-1.25$ are added to obtain

$$
d\left[p^{(1)}+r^{(1)}+r_{s}^{(1)}+r_{e}^{(1)}+r_{i}^{(1)}\right] / d t=0
$$

thus confirming the mass balance.

The proposed mechanism represents the interaction of radicals of two reacting polymers and shows how the polymer undergoing chain-end scission affects the degradation rate of the polymer undergoing random-chain scission. The degradation rate coefficient is a function of the added polymer concentration, and also depends on the temperature and pressure. This can explain the varied results found in experiments for degradation rates in polymer mixtures.

Case II. Both polymers undergo random-chain scission and interact with each other.

When polymers like polyethylene and polystyrene are present in a mixture, both polymers degrade by random-chain scission and interact with each other. We represent the reacting polymers $\mathrm{A}$ and $\mathrm{B}$ as $\mathrm{P}_{\mathrm{A}}(\mathrm{x}), \mathrm{P}_{\mathrm{B}}(\mathrm{x})$ and their radicals as $\mathrm{R}_{\mathrm{A}}{ }^{\bullet}(\mathrm{x}), \mathrm{R}_{\mathrm{B}}{ }^{\cdot}(\mathrm{x})$. Their MWDs are $\mathrm{p}_{A}(\mathrm{x}, \mathrm{t}), \mathrm{p}_{\mathrm{B}}(\mathrm{x}, \mathrm{t})$ and $\mathrm{r}_{\mathrm{A}}(\mathrm{x}, \mathrm{t}), \mathrm{r}_{\mathrm{B}}(\mathrm{x}, \mathrm{t})$ respectively. The initiation-termination reactions are

$$
\begin{gathered}
\mathrm{k}_{\mathrm{fA}} \\
\mathrm{P}_{\mathrm{A}}(\mathrm{x}) \Leftrightarrow \mathrm{R}_{\mathrm{A}} \cdot\left(\mathrm{x}^{\prime}\right)+\mathrm{R}_{\mathrm{A}} \cdot\left(\mathrm{x}-\mathrm{x}^{\prime}\right) \\
\mathrm{k}_{\mathrm{tA}} \\
\mathrm{k}_{\mathrm{fB}} \\
\mathrm{P}_{\mathrm{B}}(\mathrm{x}) \Leftrightarrow \mathrm{R}_{\mathrm{B}} \cdot\left(\mathrm{x}^{\prime}\right)+\mathrm{R}_{\mathrm{B}} \cdot\left(\mathrm{x}-\mathrm{x}^{\prime}\right) \\
\mathrm{k}_{\mathrm{tB}}
\end{gathered}
$$


The reversible hydrogen abstraction reactions are

$$
\begin{gathered}
\mathrm{k}_{\mathrm{hA}} \\
\mathrm{P}_{\mathrm{A}}(\mathrm{x}) \Leftrightarrow \mathrm{R}_{\mathrm{A}} \cdot(\mathrm{x}) \\
\mathrm{k}_{\mathrm{HA}} \\
\mathrm{k}_{\mathrm{hB}} \\
\mathrm{P}_{\mathrm{B}}(\mathrm{x}) \Leftrightarrow \mathrm{R}_{\mathrm{B}} \cdot(\mathrm{x}) \\
\mathrm{k}_{\mathrm{HB}}
\end{gathered}
$$

The depropagation reactions are

$$
\begin{gathered}
\mathrm{R}_{\mathrm{A}} \cdot \mathrm{k}_{\mathrm{bA}} \\
\mathrm{k}_{\mathrm{bB}} \\
\left.\mathrm{R}_{\mathrm{B}} \cdot \mathrm{P}_{\mathrm{A}}\left(\mathrm{x}^{\prime}\right)+\mathrm{R}_{\mathrm{A}} \cdot(\mathrm{x}) \rightarrow \mathrm{x}^{\prime}\right)
\end{gathered}
$$

The interaction between the polymers can be written as

$$
\begin{aligned}
& \mathrm{k}_{\mathrm{d}} \quad \mathrm{k}_{\mathrm{D}}
\end{aligned}
$$

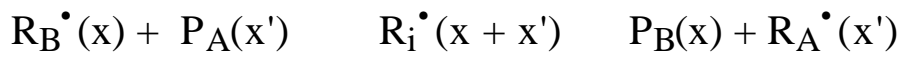

$$
\begin{aligned}
& \mathrm{k}_{\mathrm{D}} \quad \mathrm{k}_{\mathrm{d}}
\end{aligned}
$$

The population balance equations (see Table 1) are

$$
\begin{aligned}
& \partial \mathrm{p}_{\mathrm{A}} / \partial \mathrm{t}=-\mathrm{k}_{\mathrm{hA}} \mathrm{p}_{\mathrm{A}}(\mathrm{x})+\mathrm{k}_{\mathrm{HA}} \mathrm{r}_{\mathrm{A}}(\mathrm{x})+\mathrm{k}_{\mathrm{bA}} \int_{\mathrm{x}}^{\infty} \mathrm{r}_{\mathrm{A}}\left(\mathrm{x}^{\prime}\right) \Omega\left(\mathrm{x}, \mathrm{x}^{\prime}\right) \mathrm{d} \mathrm{x}^{\prime}-\mathrm{k}_{\mathrm{d}} \mathrm{p}_{\mathrm{A}} \mathrm{r}_{\mathrm{B}}{ }^{(0)} \\
& +\mathrm{k}_{\mathrm{D}} \int_{\mathrm{X}}^{\infty} \mathrm{r}_{\mathrm{i}}\left(\mathrm{x}^{\prime}\right) \Omega\left(\mathrm{x}, \mathrm{x}^{\prime}\right) \mathrm{d} \mathrm{x}^{\prime} \\
& \partial \mathrm{p}_{\mathrm{B}} / \partial \mathrm{t}=-\mathrm{k}_{\mathrm{hB}} \mathrm{p}_{\mathrm{B}}(\mathrm{x})+\mathrm{k}_{\mathrm{HB}} \mathrm{r}_{\mathrm{B}}(\mathrm{x})+\mathrm{k}_{\mathrm{bB}} \int_{\mathrm{x}}^{\infty} \mathrm{r}_{\mathrm{B}}\left(\mathrm{x}^{\prime}\right) \Omega\left(\mathrm{x}, \mathrm{x}^{\prime}\right) \mathrm{dx}^{\prime}-\mathrm{k}_{\mathrm{d}} \mathrm{p}_{\mathrm{B}} \mathrm{r}_{\mathrm{A}}{ }^{(0)} \\
& +\mathrm{k}_{\mathrm{D}} \int_{\mathrm{x}}^{\infty} \mathrm{r}_{\mathrm{i}}\left(\mathrm{x}^{\prime}\right) \Omega\left(\mathrm{x}, \mathrm{x}^{\prime}\right) \mathrm{d} \mathrm{x}^{\prime} \\
& \partial \mathrm{r}_{\mathrm{A}} / \partial \mathrm{t}=\mathrm{k}_{\mathrm{hA}} \mathrm{p}_{\mathrm{A}}(\mathrm{x})-\mathrm{k}_{\mathrm{HA}} \mathrm{r}_{\mathrm{A}}(\mathrm{x})-\mathrm{k}_{\mathrm{bA}} \mathrm{r}_{\mathrm{A}}(\mathrm{x})+\mathrm{k}_{\mathrm{bA}} \int_{\mathrm{x}}^{\infty} \mathrm{r}_{\mathrm{A}}\left(\mathrm{x}^{\prime}\right) \Omega\left(\mathrm{x}, \mathrm{x}^{\prime}\right) \mathrm{dx} \mathrm{x}^{\prime} \\
& -\mathrm{k}_{\mathrm{d}} \mathrm{p}_{\mathrm{B}}{ }^{(0)} \mathrm{r}_{\mathrm{A}}(\mathrm{x})+\mathrm{k}_{\mathrm{D}} \int_{\mathrm{x}}^{\infty} \mathrm{r}_{\mathrm{i}}\left(\mathrm{x}^{\prime}\right) \Omega\left(\mathrm{x}, \mathrm{x}^{\prime}\right) \mathrm{d} \mathrm{x}^{\prime} \\
& \partial \mathrm{r}_{\mathrm{B}} / \partial \mathrm{t}=\mathrm{k}_{\mathrm{hB}} \mathrm{p}_{\mathrm{B}}(\mathrm{x})-\mathrm{k}_{\mathrm{HB}} \mathrm{r}_{\mathrm{B}}(\mathrm{x})-\mathrm{k}_{\mathrm{bB}} \mathrm{r}_{\mathrm{B}}(\mathrm{x})+\mathrm{k}_{\mathrm{bB}} \int_{\mathrm{x}}^{\infty} \mathrm{r}_{\mathrm{B}}\left(\mathrm{x}^{\prime}\right) \Omega\left(\mathrm{x}, \mathrm{x}^{\prime}\right) \mathrm{dx} \mathrm{x}^{\prime} \\
& -\mathrm{k}_{\mathrm{d}} \mathrm{p}_{\mathrm{A}}{ }^{(0)} \mathrm{r}_{\mathrm{B}}(\mathrm{x})+\mathrm{k}_{\mathrm{D}} \int_{\mathrm{X}}^{\infty} \mathrm{r}_{\mathrm{i}}\left(\mathrm{x}^{\prime}\right) \Omega\left(\mathrm{x}, \mathrm{x}^{\prime}\right) \mathrm{d} \mathrm{x}^{\prime} \\
& \partial \mathrm{r}_{\mathrm{i}} / \partial \mathrm{t}=\mathrm{k}_{\mathrm{d}} \int_{0}^{\mathrm{x}} \mathrm{p}_{\mathrm{A}}\left(\mathrm{x}^{\prime}\right) \mathrm{r}_{\mathrm{B}}\left(\mathrm{x}-\mathrm{x}^{\prime}\right) \mathrm{d} \mathrm{x}^{\prime}+\mathrm{k}_{\mathrm{d}} \int_{0}^{\mathrm{x}} \mathrm{p}_{\mathrm{B}}\left(\mathrm{x}^{\prime}\right) \mathrm{r}_{\mathrm{A}}\left(\mathrm{x}-\mathrm{x}^{\prime}\right) \mathrm{d} \mathrm{x}^{\prime}-2 \mathrm{k}_{\mathrm{D}} \mathrm{r}_{\mathrm{i}}(\mathrm{x})
\end{aligned}
$$

Applying the moment operation, $\int_{0}^{\infty}[] \mathrm{x}^{\mathrm{n}} \mathrm{dx}$, to each mass balance equation yields

$$
\mathrm{dp}_{\mathrm{A}}{ }^{(\mathrm{n})} / \mathrm{dt}=-\mathrm{k}_{\mathrm{hA}} \mathrm{p}_{\mathrm{A}}{ }^{(\mathrm{n})}+\mathrm{k}_{\mathrm{HA}} \mathrm{r}_{\mathrm{A}}{ }^{(\mathrm{n})}+\mathrm{k}_{\mathrm{bA}} \mathrm{Z}_{\mathrm{n} 0} \mathrm{r}_{\mathrm{A}}{ }^{(\mathrm{n})}-\mathrm{k}_{\mathrm{d}} \mathrm{p}_{\mathrm{A}}{ }^{(\mathrm{n})} \mathrm{r}_{\mathrm{B}}{ }^{(0)}
$$




$$
\begin{aligned}
& +\mathrm{k}_{\mathrm{D}} \mathrm{Z}_{\mathrm{n} 0} \mathrm{r}_{\mathrm{i}}^{(\mathrm{n})} \\
& \mathrm{dp}_{\mathrm{B}}{ }^{(\mathrm{n})} / \mathrm{dt}=-\mathrm{k}_{\mathrm{hB}} \mathrm{p}_{\mathrm{B}}{ }^{(\mathrm{n})}+\mathrm{k}_{\mathrm{HB}} \mathrm{r}_{\mathrm{B}}{ }^{(\mathrm{n})}+\mathrm{k}_{\mathrm{bB}} \mathrm{Z}_{\mathrm{n} 0} \mathrm{r}_{\mathrm{B}}{ }^{(\mathrm{n})}-\mathrm{k}_{\mathrm{d}} \mathrm{p}_{\mathrm{B}}{ }^{(\mathrm{n})} \mathrm{r}_{\mathrm{A}}{ }^{(0)} \\
& +\mathrm{k}_{\mathrm{D}} \mathrm{Z}_{\mathrm{n} 0} \mathrm{r}_{\mathrm{i}}^{(\mathrm{n})}
\end{aligned}
$$

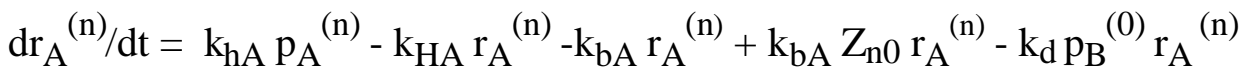

$$
\begin{aligned}
& +\mathrm{k}_{\mathrm{D}} \mathrm{Z}_{\mathrm{n} 0} \mathrm{r}_{\mathrm{i}}^{(\mathrm{n})} \\
& \mathrm{dr}_{\mathrm{B}}{ }^{(\mathrm{n})} / \mathrm{dt}=\mathrm{k}_{\mathrm{hB}} \mathrm{p}_{\mathrm{B}}{ }^{(\mathrm{n})}-\mathrm{k}_{\mathrm{HB}} \mathrm{r}_{\mathrm{A}}{ }^{(\mathrm{n})}-\mathrm{k}_{\mathrm{bB}} \mathrm{r}_{\mathrm{B}}{ }^{(\mathrm{n})}+\mathrm{k}_{\mathrm{bB}} \mathrm{Z}_{\mathrm{n} 0} \mathrm{r}_{\mathrm{B}}{ }^{(\mathrm{n})}-\mathrm{k}_{\mathrm{d}} \mathrm{p}_{\mathrm{A}}{ }^{(0)} \mathrm{r}_{\mathrm{B}}{ }^{(\mathrm{n})} \\
& +\mathrm{k}_{\mathrm{D}} \mathrm{Z}_{\mathrm{n} 0} \mathrm{r}_{\mathrm{i}}^{(\mathrm{n})}
\end{aligned}
$$

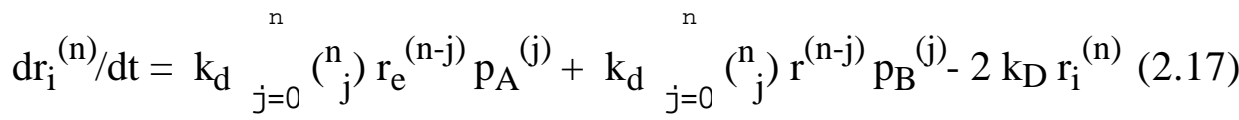

Expressions for the radical concentrations can be obtained by equating the expressions of the zeroth moments ( $\mathrm{n}=0$ in equations 2.15-2.17) to zero (QSSA). Equation 2.17 yields

$$
\mathrm{r}_{\mathrm{i}}^{(0)}=\mathrm{k}_{\mathrm{d}}\left(\mathrm{r}_{\mathrm{B}}^{(0)} \mathrm{p}_{\mathrm{A}}^{(0)}+\mathrm{r}_{\mathrm{A}}^{(0)} \mathrm{p}_{\mathrm{B}}^{(0)}\right) /\left(2 \mathrm{k}_{\mathrm{D}}\right)
$$

Equations 2.15 and 2.16 give

$$
\begin{aligned}
& \mathrm{r}_{\mathrm{A}}^{(0)}=\left(\mathrm{k}_{\mathrm{hA}} \mathrm{p}_{\mathrm{A}}{ }^{(0)}+\mathrm{k}_{\mathrm{D}} \mathrm{r}_{\mathrm{i}}^{(0)}\right) /\left(\mathrm{k}_{\mathrm{HA}}+\mathrm{k}_{\mathrm{d}} \mathrm{p}_{\mathrm{B}}{ }^{(0)}\right) \\
& \mathrm{r}_{\mathrm{B}}{ }^{(0)}=\left(\mathrm{k}_{\mathrm{hB}} \mathrm{p}_{\mathrm{B}}{ }^{(0)}+\mathrm{k}_{\mathrm{D}} \mathrm{r}_{\mathrm{i}}^{(0)}\right) /\left(\mathrm{k}_{\mathrm{HB}}+\mathrm{k}_{\mathrm{d}} \mathrm{p}_{\mathrm{A}}{ }^{(0)}\right)
\end{aligned}
$$

Substituting equations 2.18-2.20 in equations 2.13 and 2.14 yields

$$
\begin{aligned}
& \mathrm{dp}_{\mathrm{A}}{ }^{(0)} / \mathrm{dt}=\mathrm{k}_{\mathrm{bA}} \mathrm{r}_{\mathrm{A}}{ }^{(0)} \\
& \mathrm{dp}_{\mathrm{B}}{ }^{(0)} / \mathrm{dt}=\mathrm{k}_{\mathrm{bB}} \mathrm{r}_{\mathrm{B}}{ }^{0)}
\end{aligned}
$$

Expressions for $r_{A}{ }^{(0)}$ and $r_{B}{ }^{(0)}$ can be obtained by solving equations 2.19 and 2.20 simultaneously with equation 2.18. Equations 2.21 and 2.22 show how the degradation rate of a polymer depends on the concentration of the other polymer in the mixture. The degradation rate coefficient is a function of the added polymer concentration, and the experimental conditions (temperature and pressure).

Case III. Both polymers undergo chain-end scission and interact with each other.

Certain polymers like PAMS undergo only chain-end scission. In cases where both polymers, A and B, degrade by chain-end scission and interact with each other, the 
radical reactions for polymers can be written based on the Rice-Herzfeld mechanism. The polymers A and B, the chain-end radical, the specific radical, and the specific product are represented as $\mathrm{P}_{\mathrm{A}}(\mathrm{x}), \mathrm{R}_{\mathrm{eA}}{ }^{\bullet}(\mathrm{x}), \mathrm{R}_{\mathrm{SA}}{ }^{\bullet}(\mathrm{x}), \mathrm{Q}_{\mathrm{sA}}\left(\mathrm{x}_{\mathrm{SA}}\right)$ and $\mathrm{P}_{\mathrm{B}}(\mathrm{x}), \mathrm{R}_{\mathrm{eB}}{ }^{\bullet}(\mathrm{x}), \mathrm{R}_{\mathrm{sB}}{ }^{\bullet}(\mathrm{x}), \mathrm{Q}_{\mathrm{sB}}\left(\mathrm{x}_{\mathrm{sB}}\right)$, respectively. The formation of chain-end radicals by a reversible random-scission initiationtermination reaction is

$$
\begin{gathered}
\mathrm{P}_{\mathrm{A}}(\mathrm{x}) \underset{\mathrm{fs} A}{\mathrm{k}_{\mathrm{tsA}}} \Leftrightarrow \mathrm{R}_{\mathrm{eA}} \cdot(\mathrm{x})+\mathrm{R}_{\mathrm{eA}} \cdot\left(\mathrm{x}-\mathrm{x}^{\prime}\right) \\
\mathrm{k}_{\mathrm{fsB}} \\
\mathrm{P}_{\mathrm{B}}(\mathrm{x}) \underset{\mathrm{k}}{\Leftrightarrow} \mathrm{R}_{\mathrm{eB}} \cdot(\mathrm{x})+\mathrm{R}_{\mathrm{eB}} \cdot\left(\mathrm{x}-\mathrm{x}^{\prime}\right)
\end{gathered}
$$

Hydrogen abstraction by the chain-end radical is considered reversible,

$$
\begin{aligned}
\mathrm{P}_{\mathrm{A}}(\mathrm{x}) \underset{\mathrm{keA}}{\Leftrightarrow} \mathrm{R}_{\mathrm{eA}} \cdot(\mathrm{x}) \\
\mathrm{k}_{\mathrm{HeA}} \\
\mathrm{k}_{\mathrm{heB}} \\
\mathrm{P}_{\mathrm{B}}(\mathrm{x}) \stackrel{\mathrm{k}_{\mathrm{eB}}}{\Leftrightarrow} \mathrm{k}_{\mathrm{HeB}}(\mathrm{x})
\end{aligned}
$$

The chain-end radical can undergo radical isomerization via a cyclic transition state to form a specific radical,

$$
\begin{aligned}
& \mathrm{k}_{\mathrm{ihA}} \\
& \mathrm{R}_{\mathrm{eA}}{ }^{\bullet}(\mathrm{x}) \Leftrightarrow \mathrm{R}_{\mathrm{SA}}{ }^{\bullet}(\mathrm{x}) \\
& \mathrm{k}_{\mathrm{iHA}} \\
& \mathrm{k}_{\mathrm{ihB}}
\end{aligned}
$$

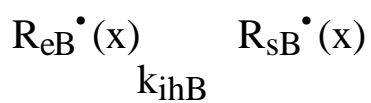

The depropagation reaction yields the specific product and a chain-end radical from a specific radical

$$
\begin{aligned}
& \mathrm{k}_{\mathrm{bsA}} \\
& \mathrm{R}_{\mathrm{SA}}{ }^{\bullet}(\mathrm{x}) \underset{\mathrm{k}_{\mathrm{bsB}}}{\rightarrow} \mathrm{Q}_{\mathrm{sA}}\left(\mathrm{x}_{\mathrm{S}}\right)+\mathrm{R}_{\mathrm{eA}} \cdot\left(\mathrm{x}-\mathrm{x}_{\mathrm{S}}\right) \\
& \mathrm{R}_{\mathrm{SB}}{ }^{\bullet}(\mathrm{x}) \rightarrow \mathrm{Q}_{\mathrm{sB}}\left(\mathrm{x}_{\mathrm{S}}\right)+\mathrm{R}_{\mathrm{eB}}{ }^{\bullet}\left(\mathrm{x}-\mathrm{x}_{\mathrm{S}}\right)
\end{aligned}
$$


where $\mathrm{x}_{\mathrm{SA}}$ and $\mathrm{x}_{\mathrm{SB}}$ are the MWs of the specific products of polymer $\mathrm{A}$ and $\mathrm{B}$, respectively. The interaction of the two polymers is given by the disproportionation reaction

$$
\begin{array}{rc}
\mathrm{k}_{\mathrm{d}} & \mathrm{k}_{\mathrm{D}} \\
\mathrm{R}_{\mathrm{eB}} \cdot(\mathrm{x})+\mathrm{P}_{\mathrm{A}}\left(\mathrm{x}^{\prime}\right) & \Leftrightarrow \mathrm{R}_{\mathrm{i}}\left(\mathrm{x}+\mathrm{x}^{\prime}\right) \Leftrightarrow \mathrm{P}_{\mathrm{B}}(\mathrm{x})+\mathrm{R}_{\mathrm{eA}}{ }^{\bullet}\left(\mathrm{x}^{\prime}\right) \\
\mathrm{k}_{\mathrm{D}} & \mathrm{k}_{\mathrm{d}}
\end{array}
$$

The population balance equations are

$$
\begin{aligned}
& \partial \mathrm{p}_{\mathrm{A}} / \partial \mathrm{t}=-\mathrm{k}_{\mathrm{heA}} \mathrm{p}_{\mathrm{A}}(\mathrm{x})+\mathrm{k}_{\mathrm{HeA}} \mathrm{r}_{\mathrm{eA}}(\mathrm{x})-\mathrm{k}_{\mathrm{d}} \mathrm{p}_{\mathrm{A}}(\mathrm{x}) \mathrm{r}_{\mathrm{eB}}{ }^{(0)} \\
& +\mathrm{k}_{\mathrm{D}} \int_{\mathrm{X}}^{\infty} \mathrm{r}_{\mathrm{i}}\left(\mathrm{x}^{\prime}\right) \Omega\left(\mathrm{x}, \mathrm{x}^{\prime}\right) \mathrm{d} \mathrm{x}^{\prime} \\
& \partial \mathrm{p}_{\mathrm{B}} / \partial \mathrm{t}=-\mathrm{k}_{\mathrm{heB}} \mathrm{p}_{\mathrm{B}}(\mathrm{x})+\mathrm{k}_{\mathrm{HeB}} \mathrm{r}_{\mathrm{eB}}(\mathrm{x})-\mathrm{k}_{\mathrm{d}} \mathrm{p}_{\mathrm{B}}(\mathrm{x}) \mathrm{r}_{\mathrm{eA}}{ }^{(0)} \\
& +\mathrm{k}_{\mathrm{D}} \int_{\mathrm{X}}^{\infty} \mathrm{r}_{\mathrm{i}}\left(\mathrm{x}^{\prime}\right) \Omega\left(\mathrm{x}, \mathrm{x}^{\prime}\right) \mathrm{dx} \mathrm{x}^{\prime} \\
& \partial \mathrm{q}_{\mathrm{sA}} / \partial \mathrm{t}=\mathrm{k}_{\mathrm{bsA}} \int_{\mathrm{x}}^{\infty} \mathrm{r}_{\mathrm{sA}}\left(\mathrm{x}^{\prime}\right) \delta\left(\mathrm{x}^{\prime}-\mathrm{x}_{\mathrm{S}}\right) \mathrm{dx} \mathrm{x}^{\prime} \\
& \partial \mathrm{q}_{\mathrm{sB}} / \partial \mathrm{t}=\mathrm{k}_{\mathrm{bsB}} \int_{\mathrm{x}}^{\infty} \mathrm{r}_{\mathrm{sA}}\left(\mathrm{x}^{\prime}\right) \delta\left(\mathrm{x}^{\prime}-\mathrm{x}_{\mathrm{s}}\right) \mathrm{dx} \\
& \partial \mathrm{r}_{\mathrm{sA}} / \partial \mathrm{t}=\mathrm{k}_{\mathrm{ihA}} \mathrm{r}_{\mathrm{eA}}(\mathrm{x})-\mathrm{k}_{\mathrm{iHA}} \mathrm{r}_{\mathrm{sA}}(\mathrm{x})-\mathrm{k}_{\mathrm{bsA}} \mathrm{r}_{\mathrm{sA}}(\mathrm{x}) \\
& \partial \mathrm{r}_{\mathrm{sB}} / \partial \mathrm{t}=\mathrm{k}_{\mathrm{ihB}} \mathrm{r}_{\mathrm{eB}}(\mathrm{x})-\mathrm{k}_{\mathrm{iHB}} \mathrm{r}_{\mathrm{sB}}(\mathrm{x})-\mathrm{k}_{\mathrm{bsB}} \mathrm{r}_{\mathrm{sB}}(\mathrm{x}) \\
& \partial \mathrm{r}_{\mathrm{eA}} / \partial \mathrm{t}=\mathrm{k}_{\mathrm{heA}} \mathrm{p}_{\mathrm{A}}(\mathrm{x})-\mathrm{k}_{\mathrm{HeA}} \mathrm{r}_{\mathrm{eA}}(\mathrm{x})-\mathrm{k}_{\mathrm{ihA}} \mathrm{r}_{\mathrm{eA}}(\mathrm{x})+\mathrm{k}_{\mathrm{iHA}} \mathrm{r}_{\mathrm{sA}}(\mathrm{x}) \\
& +\mathrm{k}_{\mathrm{bs}} \int_{\mathrm{x}}^{\infty} \mathrm{r}_{\mathrm{sA}}\left(\mathrm{x}^{\prime}\right) \delta\left[\mathrm{x}-\left(\mathrm{x}^{\prime}-\mathrm{x}_{\mathrm{S}}\right)\right] \mathrm{dx^{ \prime }}-\mathrm{k}_{\mathrm{d}} \mathrm{p}_{\mathrm{B}}{ }^{(0)} \mathrm{r}_{\mathrm{eA}}(\mathrm{x}) \\
& +\mathrm{k}_{\mathrm{D}} \int_{\mathrm{x}}^{\infty} \mathrm{r}_{\mathrm{i}}\left(\mathrm{x}^{\prime}\right) \Omega\left(\mathrm{x}, \mathrm{x}^{\prime}\right) \mathrm{d} \mathrm{x}^{\prime} \\
& \partial \mathrm{r}_{\mathrm{eB}} / \partial \mathrm{t}=\mathrm{k}_{\mathrm{heB}} \mathrm{p}_{\mathrm{B}}(\mathrm{x})-\mathrm{k}_{\mathrm{HeB}} \mathrm{r}_{\mathrm{eB}}(\mathrm{x})-\mathrm{k}_{\mathrm{ihB}} \mathrm{r}_{\mathrm{eB}}(\mathrm{x})+\mathrm{k}_{\mathrm{iHB}} \mathrm{r}_{\mathrm{sB}}(\mathrm{x})
\end{aligned}
$$

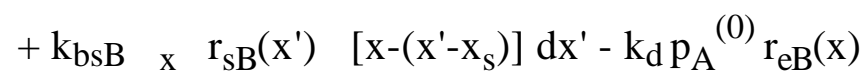

$$
\begin{aligned}
& +\mathrm{k}_{\mathrm{D}} \int_{\mathrm{x}}^{\infty} \mathrm{r}_{\mathrm{i}}\left(\mathrm{x}^{\prime}\right) \Omega\left(\mathrm{x}, \mathrm{x}^{\prime}\right) \mathrm{d} \mathrm{x}^{\prime} \\
& \partial \mathrm{r}_{\mathrm{i}} / \partial \mathrm{t}=\mathrm{k}_{\mathrm{d}} \int_{0}^{\mathrm{x}} \mathrm{p}_{\mathrm{A}}\left(\mathrm{x}^{\prime}\right) \mathrm{r}_{\mathrm{eB}}\left(\mathrm{x}-\mathrm{x}^{\prime}\right) \mathrm{d} \mathrm{x}^{\prime}+\mathrm{k}_{\mathrm{d}} \int_{0}^{\mathrm{x}} \mathrm{p}_{\mathrm{B}}\left(\mathrm{x}^{\prime}\right) \mathrm{r}_{\mathrm{eA}}\left(\mathrm{x}-\mathrm{x}^{\prime}\right) \mathrm{d} \mathrm{x}^{\prime}-2 \mathrm{k}_{\mathrm{D}} \mathrm{r}_{\mathrm{i}}(\mathrm{x})
\end{aligned}
$$

Applying the moment operation, $\int_{0}^{\infty}[] \mathrm{x}^{\mathrm{n}} \mathrm{dx}$, to each mass balance equation yields

$$
\begin{aligned}
& \mathrm{dp}_{\mathrm{A}}{ }^{(\mathrm{n})} / \mathrm{dt}=-\mathrm{k}_{\mathrm{heA}} \mathrm{p}_{\mathrm{A}}{ }^{(\mathrm{n})}+\mathrm{k}_{\mathrm{HeA}} \mathrm{r}_{\mathrm{eA}}{ }^{(\mathrm{n})}-\mathrm{k}_{\mathrm{d}} \mathrm{p}_{\mathrm{A}}{ }^{(\mathrm{n})} \mathrm{r}_{\mathrm{eB}}{ }^{(0)}+\mathrm{k}_{\mathrm{D}} \mathrm{Z}_{\mathrm{n} 0} \mathrm{r}_{\mathrm{i}}{ }^{(\mathrm{n})} \\
& \mathrm{dp}_{\mathrm{B}}{ }^{(\mathrm{n})} / \mathrm{dt}=-\mathrm{k}_{\mathrm{heB}} \mathrm{p}_{\mathrm{B}}{ }^{(\mathrm{n})}+\mathrm{k}_{\mathrm{HeB}} \mathrm{r}_{\mathrm{eB}}{ }^{(\mathrm{n})}-\mathrm{k}_{\mathrm{d}} \mathrm{p}_{\mathrm{B}}{ }^{(\mathrm{n})} \mathrm{r}_{\mathrm{eA}}{ }^{(0)}+\mathrm{k}_{\mathrm{D}} \mathrm{Z}_{\mathrm{n} 0} \mathrm{r}_{\mathrm{i}}{ }^{(\mathrm{n})}
\end{aligned}
$$




$$
\begin{aligned}
& \mathrm{dq}_{\mathrm{sA}}{ }^{(\mathrm{n})} / \mathrm{dt}=\mathrm{k}_{\mathrm{bsA}} \mathrm{x}_{\mathrm{sA}}{ }^{\mathrm{n}} \mathrm{r}_{\mathrm{sA}}{ }^{(0)} \\
& \mathrm{dq}_{\mathrm{sB}}{ }^{(\mathrm{n})} / \mathrm{dt}=\mathrm{k}_{\mathrm{bsB}} \mathrm{x}_{\mathrm{sB}}{ }^{\mathrm{n}} \mathrm{r}_{\mathrm{sB}}{ }^{(0)} \\
& \mathrm{dr}_{\mathrm{SA}}{ }^{(\mathrm{n})} / \mathrm{dt}=\mathrm{k}_{\mathrm{ihA}} \mathrm{r}_{\mathrm{eA}}{ }^{(\mathrm{n})}-\mathrm{k}_{\mathrm{iHA}} \mathrm{r}_{\mathrm{SA}}{ }^{(\mathrm{n})}-\mathrm{k}_{\mathrm{bsA}} \mathrm{r}_{\mathrm{sA}}{ }^{(\mathrm{n})} \\
& \mathrm{dr}_{\mathrm{sB}}{ }^{(\mathrm{n})} / \mathrm{dt}=\mathrm{k}_{\mathrm{ihB}} \mathrm{r}_{\mathrm{eB}}{ }^{(\mathrm{n})}-\mathrm{k}_{\mathrm{iHB}} \mathrm{r}_{\mathrm{sB}}{ }^{(\mathrm{n})}-\mathrm{k}_{\mathrm{bsB}} \mathrm{r}_{\mathrm{sB}}{ }^{(\mathrm{n})} \\
& \mathrm{dr}_{\mathrm{eA}}{ }^{(\mathrm{n})} / \mathrm{dt}=\mathrm{k}_{\mathrm{heA}} \mathrm{p}_{\mathrm{A}}^{(\mathrm{n})}-\mathrm{k}_{\mathrm{HeA}} \mathrm{r}_{\mathrm{eA}}{ }^{(\mathrm{n})}-\mathrm{k}_{\mathrm{ihA}} \mathrm{r}_{\mathrm{eA}}{ }^{(\mathrm{n})}+\mathrm{k}_{\mathrm{iHA}} \mathrm{r}_{\mathrm{SA}}{ }^{(\mathrm{n})} \\
& +\mathrm{k}_{\mathrm{bsA}} \sum_{j=0}^{\mathrm{n}}\left({ }_{\mathrm{j}}^{\mathrm{n}}\right)\left(\mathrm{x}_{\mathrm{SA}}\right)^{\mathrm{j}}(-1)^{\mathrm{j}} \mathrm{r}_{\mathrm{sA}}{ }^{(\mathrm{n}-\mathrm{j})}-\mathrm{k}_{\mathrm{d}} \mathrm{p}_{\mathrm{B}}{ }^{(0)} \mathrm{r}_{\mathrm{eA}}{ }^{(\mathrm{n})}+\mathrm{k}_{\mathrm{D}} \mathrm{Z}_{\mathrm{n} 0} \mathrm{r}_{\mathrm{i}}{ }^{(\mathrm{n})} \\
& \mathrm{dr}_{\mathrm{eB}}{ }^{(\mathrm{n})} / \mathrm{dt}=\mathrm{k}_{\mathrm{heB}} \mathrm{p}_{\mathrm{B}}{ }^{(\mathrm{n})}-\mathrm{k}_{\mathrm{HeB}} \mathrm{r}_{\mathrm{eB}}{ }^{(\mathrm{n})}-\mathrm{k}_{\mathrm{ihB}} \mathrm{r}_{\mathrm{eB}}{ }^{(\mathrm{n})}+\mathrm{k}_{\mathrm{iHB}} \mathrm{r}_{\mathrm{sB}}{ }^{(\mathrm{n})} \\
& +k_{b s B} \sum_{j=0}^{n}\left({ }_{j}^{n}\right)\left(x_{s B}\right)^{j}(-1)^{j} r_{s B}{ }^{(n-j)}-k_{d} p_{A}{ }^{(0)} r_{e B}{ }^{(n)}+k_{D} Z_{n 0} r_{i}{ }^{(n)} \\
& d r_{i}^{(n)} / d t=k_{d} \sum_{j=0}^{n}\left({ }_{j}^{n}\right) r_{e}^{(n-j)} p_{A}^{(j)}+k_{d} \sum_{j=0}^{n}\left({ }_{j}^{n}\right) r^{(n-j)} p_{B}{ }^{(j)}-2 k_{D} r_{i}^{(n)}
\end{aligned}
$$

Expressions for the radical concentrations can be obtained by equating the expressions of the zeroth moments ( $\mathrm{n}=0$ in equations 3.23-3.27) to zero. Equations 3.23 and 3.24 yield

$$
\begin{aligned}
& \mathrm{r}_{\mathrm{sA}}{ }^{(0)}=\mathrm{r}_{\mathrm{eA}}{ }^{(0)} \mathrm{k}_{\mathrm{ihA}} /\left(\mathrm{k}_{\mathrm{bsA}}+\mathrm{k}_{\mathrm{iHA}}\right) \\
& \mathrm{r}_{\mathrm{sB}}{ }^{(0)}=\mathrm{r}_{\mathrm{eB}}{ }^{(0)} \mathrm{k}_{\mathrm{ihB}} /\left(\mathrm{k}_{\mathrm{bsB}}+\mathrm{k}_{\mathrm{iHB}}\right)
\end{aligned}
$$

Equating equations 3.25 and 3.26 to zero gives

$$
\begin{aligned}
& \mathrm{r}_{\mathrm{eA}}{ }^{(0)}=\left(\mathrm{k}_{\mathrm{D}} \mathrm{r}_{\mathrm{i}}^{(0)}+\mathrm{k}_{\mathrm{heA}} \mathrm{p}_{\mathrm{A}}{ }^{(0)}\right) /\left(\mathrm{k}_{\mathrm{d}} \mathrm{p}_{\mathrm{B}}{ }^{(0)}+\mathrm{k}_{\mathrm{heA}}\right) \\
& \mathrm{r}_{\mathrm{eB}}{ }^{(0)}=\left(\mathrm{k}_{\mathrm{D}} \mathrm{r}_{\mathrm{i}}^{(0)}+\mathrm{k}_{\mathrm{heB}} \mathrm{p}_{\mathrm{B}}^{(0)}\right) /\left(\mathrm{k}_{\mathrm{d}} \mathrm{p}_{\mathrm{A}}{ }^{(0)}+\mathrm{k}_{\mathrm{heB}}\right)
\end{aligned}
$$

where, by equation 3.27 ,

$$
\mathrm{r}_{\mathrm{i}}^{(0)}=\mathrm{k}_{\mathrm{d}}\left(\mathrm{r}_{\mathrm{eB}}{ }^{(0)} \mathrm{p}_{\mathrm{A}}{ }^{(0)}+\mathrm{r}_{\mathrm{eA}}{ }^{(0)} \mathrm{p}_{\mathrm{B}}^{(0)}\right) /\left(2 \mathrm{k}_{\mathrm{D}}\right)
$$

Using these expressions in equations 3.19 and 3.20 yields

$$
\begin{aligned}
& \mathrm{dp}_{\mathrm{A}}{ }^{(0)} / \mathrm{dt}=0 \\
& \mathrm{dp}_{\mathrm{B}}{ }^{(0)} / \mathrm{dt}=0
\end{aligned}
$$

and therefore,

$$
\mathrm{p}_{\mathrm{A}}{ }^{(0)}=\mathrm{p}_{\mathrm{A} 0}{ }^{(0)}, \mathrm{p}_{\mathrm{B}}{ }^{(0)}=\mathrm{p}_{\mathrm{B} 0}{ }^{(0)}
$$


This is consistent with chain-end scission kinetics (Madras et al., 1996b), where the zeroth moment (molar concentration) of the polymer is a constant. Equations 3.21 and 3.22 are

$$
\begin{aligned}
& \mathrm{dq}_{\mathrm{sA}}{ }^{(0)} / \mathrm{dt}=\mathrm{k}_{\mathrm{bsA}} \mathrm{r}_{\mathrm{sA}}{ }^{(0)} \\
& \mathrm{dq}_{\mathrm{sB}}{ }^{(0)} / \mathrm{dt}=\mathrm{k}_{\mathrm{bsB}} \mathrm{r}_{\mathrm{sB}}{ }^{(0)}
\end{aligned}
$$

Expressions for $\mathrm{r}_{\mathrm{SA}}{ }^{(0)}$ and $\mathrm{r}_{\mathrm{SB}}{ }^{(0)}$ can be obtained by simultaneously solving equations 3.30 and 3.31 with equation 3.32. Equations 3.36 and 3.37 are coupled ordinary differential equations that can be solved numerically. Analytical algebraic solutions are possible for special value of the rate parameters. The equations show how the degradation rate of a polymer depends on the concentration of the other polymer in the mixture. The degradation rate coefficient is also a function of temperature and pressure.

\section{Results and Discussion}

Polymers thermolytically degrade by chain-end scission and/or random-chain scission. The polymer interaction is complex and is influenced by the experimental conditions and polymer type. For example, Roy et al. (1978) observed no interaction between polystyrene and polyethylene at pressures higher than $200 \mathrm{~mm} \mathrm{Hg}$ but observed interaction at lower pressures. McCaffrey et al. (1993) observed a significant increase in the thermolytic degradation rate of polyethylene due to the presence of polystyrene. They proposed that the interaction was due to hydrogen abstraction from polyethylene by polystyrene radicals. Gardner et al. (1993) observed an eight fold increase in the pyrolytic chain-end scission rate of polystyrene at $430{ }^{\circ} \mathrm{C}$ in the presence of poly(methyl acrylate) (PMA) or poly(butyl acrylate) (PBA), while the chain-end degradation rates of PBA and PMA decreased eight fold. However, PBA or PMA did not affect PAMS degradation rate. They also concluded that hydrogen abstraction plays an important role in polymer interactions.

We have proposed degradation mechanisms for three common types of polymer degradation with interactions by a disproportionation reaction for hydrogen abstraction. The degradation rate equations (Equations 1.32, 1.34, 2.19, 2.20, 3.36 and 3.37) for 
binary mixtures show that, depending on the particular polymer, another polymer in the mixture can increase, decrease, or have no effect on degradation rate. As shown by the above equations, the interaction would depend on the fundamental radical rate parameters (and thus temperature and pressure) and the concentration of the polymers in the mixture.

As an example, we have measured the influence of PAMS mass concentration on polystyrene degradation at $275^{\circ} \mathrm{C}$. Since polystyrene degrades by random-chain scission and PAMS degrades by chain-end scission, the polystyrene degradation rate is given by equation 1.34. If the rates of the elementary steps are known, this equation can be solved numerically. However, reasonable assumptions allow us to obtain an analytical solution for the polystyrene degradation rate.

The hydrogen abstraction rate by the radicals in equation 1.5 is negligible compared to the hydrogen abstraction in equation 1.8. Thus, $\mathrm{k}_{\mathrm{He}}=0$ and with equation 1.33 , equation 1.31 is

$$
\mathrm{r}_{\mathrm{e}}^{(0)}=\mathrm{p}_{\mathrm{B} 0}{ }^{(0)}\left(2 \mathrm{k}_{\mathrm{he}} \mathrm{k}_{\mathrm{H}}+\mathrm{k}_{\mathrm{d}}\left(\mathrm{k}_{\mathrm{he}}+\mathrm{k}_{\mathrm{h}}\right) \mathrm{p}_{\mathrm{A}}{ }^{(0)}\right) /\left(\mathrm{k}_{\mathrm{d}} \mathrm{k}_{\mathrm{H}} \mathrm{p}_{\mathrm{A}}{ }^{(0)}\right)
$$

The hydrogen abstraction in equation 1.8 is more significant than the hydrogen abstraction rate in equation 1.2. Thus, we assume

$$
\mathrm{k}_{\mathrm{H}} \ll \mathrm{k}_{\mathrm{d}} \mathrm{p}_{\mathrm{A}}{ }^{(0)} / 2
$$

Equation 4.1 becomes

$$
\mathrm{r}_{\mathrm{e}}^{(0)}=\mathrm{p}_{\mathrm{B} 0}^{(0)}\left(\mathrm{k}_{\mathrm{he}}+\mathrm{k}_{\mathrm{h}}\right) / \mathrm{k}_{\mathrm{H}}
$$

Substituting equations 4.3 and 1.30 in equation 1.34 yields

$$
\mathrm{dp}_{\mathrm{A}}{ }^{(0)} / \mathrm{dt}=\mathrm{k}_{\mathrm{r}} \mathrm{p}_{\mathrm{A}}{ }^{(0)}
$$

whose solution is

$$
\mathrm{p}_{\mathrm{A}}{ }^{(0)}(\mathrm{t})=\mathrm{p}_{\mathrm{A} 0}{ }^{(0)} \exp \left(\mathrm{k}_{\mathrm{r}} \mathrm{t}\right)
$$

and a plot of $\ln \left(\mathrm{p}^{(0)} / \mathrm{p}_{0}{ }^{(0)}\right)$ is linear in time with slope $\mathrm{k}_{\mathrm{r}}$,

$$
\mathrm{k}_{\mathrm{r}}=\left(2 \mathrm{k}_{\mathrm{b}} \mathrm{k}_{\mathrm{h}}+\left(2 \mathrm{k}_{\mathrm{b}} \mathrm{k}_{\mathrm{d}}\left(\mathrm{k}_{\mathrm{he}}+\mathrm{k}_{\mathrm{h}}\right) \mathrm{p}_{\mathrm{B} 0}{ }^{(0)} / \mathrm{k}_{\mathrm{H}}\right)\right) /\left(2 \mathrm{k}_{\mathrm{H}}+\mathrm{k}_{\mathrm{d}} \mathrm{p}_{\mathrm{B} 0}{ }^{(0)}\right)
$$

Similar to equation 4.2,

$$
\mathrm{k}_{\mathrm{H}} \ll<\mathrm{k}_{\mathrm{d}} \mathrm{p}_{\mathrm{B} 0}{ }^{(0)} / 2
$$


and equation 4.6 yields

$$
\begin{aligned}
\mathrm{k}_{\mathrm{r}} & =\left(2 \mathrm{k}_{\mathrm{b}} \mathrm{k}_{\mathrm{h}} /\left(\mathrm{k}_{\mathrm{d}} \mathrm{p}_{\mathrm{B} 0}{ }^{(0)}\right)\right)+\left(\mathrm{k}_{\mathrm{b}}\left(\mathrm{k}_{\mathrm{he}}+\mathrm{k}_{\mathrm{h}}\right) / \mathrm{k}_{\mathrm{H}}\right) \\
& =\left(2 \mathrm{k}_{\mathrm{b}} \mathrm{k}_{\mathrm{h}} \mathrm{M}_{\mathrm{B} 0} /\left(\mathrm{k}_{\mathrm{d}} \mathrm{p}_{\mathrm{B} 0}{ }^{(1)}\right)\right)+\left(\mathrm{k}_{\mathrm{b}}\left(\mathrm{k}_{\mathrm{he}}+\mathrm{k}_{\mathrm{h}}\right) / \mathrm{k}_{\mathrm{H}}\right)
\end{aligned}
$$

Here, $\mathrm{p}_{\mathrm{B} 0}{ }^{(1)}$ and $\mathrm{M}_{\mathrm{B} 0}$ are the initial mass concentration and the number-average molecular weight, respectively, of PAMS.

The random-scission degradation rate coefficient, $\mathrm{k}_{\mathrm{r}}$, was determined from the experimental data by analyzing the time dependence of the polymer-mixture MWDs. Because the mass of specific products formed by polystyrene chain-end scission at $275^{\circ} \mathrm{C}$ for 10 hours is less than 2\% (Madras et al., 1996c), we consider that polystyrene degrades solely by random-chain scission .

Polystyrene degrades rapidly at low reaction times due to weak links in the polymer chain caused by side-group asymmetry or chain-branching (Chiantore et al., 1981; Madras et al., 1996c). The weak and strong links in polystyrene can be represented by additive distributions, so that the total molar concentration, $\mathrm{p}_{\text {Atot }}{ }^{(0)}$, of the polymer is the sum of the molar concentrations of the weak, $\mathrm{p}_{\mathrm{Aw}}{ }^{(0)}$, and strong links, $\mathrm{p}_{\mathrm{A}}{ }^{(0)}$ (Madras et al., 1996c). As the weak link concentration is approximately two orders of magnitude smaller than the strong link concentration, only the random rate coefficients of strong links are examined in this study. The initial molar concentration of the strong links in polystyrene, $\mathrm{p}_{\mathrm{A} 0}{ }^{(0)}$, is determined from the intercept of the regressed line of the $\mathrm{p}_{\text {Atot }}{ }^{(0)} / \mathrm{p}_{\text {Atot } 0}{ }^{(0)}$ data for $\mathrm{t} \geq 45$ minutes. The slopes, corresponding to the rate coefficient for random scission, $\mathrm{k}_{\mathrm{r}}$, are determined from the plot of $\ln \left(\mathrm{p}_{\mathrm{A}}{ }^{(0)} / \mathrm{p}_{\mathrm{A} 0}{ }^{(0)}\right)$ versus time, as given by equation 4.5 (Figure 3). The line for zero concentration of PAMS is curved slightly due to a MW-dependent rate. Madras et al. (1997) show how data are analyzed when the polystyrene degradation rate coefficient is a function of $\mathrm{MW}$, i.e., $\mathrm{k}_{\mathrm{r}}(\mathrm{x})$. Equation 1.34 explains how the polystyrene degradation rate coefficient depends on PAMS concentration. Only zeroth moments (molar concentrations) are required to derive this key relationship. The polystyrene degradation rate coefficient, $\mathrm{k}_{\mathrm{r}}$, given by equation 4.9 decreases with 
increasing PAMS mass (or molar) concentration. This is consistent with the experimental data (inset of Figure 4).

Ratios of the rate parameters can be determined by rewriting equation 4.9 ,

$$
\mathrm{k}_{\mathrm{r}}=\mathrm{k}_{1}+\left(\mathrm{k}_{2} / \mathrm{p}_{\mathrm{B} 0}{ }^{(1)}\right)
$$

where $\mathrm{k}_{1}=\mathrm{k}_{\mathrm{b}}\left(\mathrm{k}_{\mathrm{he}}+\mathrm{k}_{\mathrm{h}}\right) / \mathrm{k}_{\mathrm{H}}$ and $\mathrm{k}_{2}=2 \mathrm{k}_{\mathrm{b}} \mathrm{k}_{\mathrm{h}} \mathrm{M}_{\mathrm{B} 0} / \mathrm{k}_{\mathrm{d}}$. The slope and intercept of $\mathrm{k}_{\mathrm{r}}$

versus $1 / \mathrm{p}_{\mathrm{B} 0}{ }^{(1)}$ (Figure 4) yield the rate parameters, $\mathrm{k}_{1}\left(=0.003 \mathrm{~min}^{-1}\right)$ and $\mathrm{k}_{2}(=0.006 \mathrm{~g}$

$\left.\mathrm{L}^{-1} \min ^{-1}\right)$. For $\mathrm{p}_{\mathrm{B} 0}^{(1)}=0$, equation 4.6 gives $\mathrm{k}_{\mathrm{r}}=\mathrm{k}_{\mathrm{b}} \mathrm{k}_{\mathrm{h}} / \mathrm{k}_{\mathrm{H}}\left(=0.013 \mathrm{~min}^{-1}\right.$ by experimental data).

The hypothesized interaction of the degrading polymers is through the free radicals and their rates of hydrogen abstraction. When $\mathrm{k}_{\mathrm{d}}=\mathrm{k}_{\mathrm{D}}=0$, the two polymers react independently and the moment equations 1.19-1.25, are identical to those derived for a single polymer undergoing chain-end scission or random-chain scission (Madras and McCoy, 1997). The rate coefficient for random-chain scission of polystyrene is a function of the PAMS mixture concentration through the fundamental radical rate parameters, $\mathrm{k}_{\mathrm{b}}$, $\mathrm{k}_{\mathrm{h}}, \mathrm{k}_{\mathrm{he}}, \mathrm{k}_{\mathrm{d}}, \mathrm{k}_{\mathrm{H}}$, and the initial number-average molecular weight of PAMS (equation 4.10). The addition of PAMS inhibits the random-chain scission of polystyrene, similar to the effect on hydrogen-donors on the degradation of polystyrene (Madras and McCoy, 1997). The results suggest that the interaction between polymers is solely due to hydrogen abstraction.

\section{Acknowledgments}

The financial support of Pittsburgh Energy Technology Center Grant No. DOE DEFG22-94PC94204 and EPA Grant No. CR 822990-01-0 is gratefully acknowledged. The authors thank Professor J.M. Smith for helpful discussions and Dr. Yoichi Kodera for analyzing the mineral oil sample (Figure 2). 


\section{References}

Chiantore, O.; Camino, G.; Costa, L.; Grassie, N., "Weak Links in Polystyrene," Poly. Deg. and Stab., 3, 209 (1981).

Clough, R.L.; Billingham, N.C.; Gillen, K.T., Polymer Durability, ACS Symposium Series (1996).

Gardner, P.; R. Lehrle; D. Turner, Polymer Degradation Modified by Blending with Polymers Chosen on the Basis of Their Ф-Factors," J. Anal. Appl. Pyr., 25, 11 (1993).

Gavalas, G. R., "The Long Chain Approximation in Free Radical Reaction Systems, " Chem. Eng. Sci., 21, 133 (1966).

Koo, J.K., S.W. Kim, Y. H.. Seo, "Characterization of Aromatic Hydrocarbon Formation From Pyrolysis of Polyethylene-Polystyrene Mixtures," Resources, Conservation and Recycling, 5, 365 (1991).

Koo, J.K., S.W. Kim, "Reaction Kinetic Model for Optimal Pyrolysis of Plastic Waste Mixtures," Waste Management and Research, 11, 515 (1993).

Madras, G., J.M. Smith, B.J. McCoy, "Effect of Tetralin on the Degradation of Polymer in Solution," I\&EC Research, 34, 4222 (1995).

Madras, G., J.M. Smith, B.J. McCoy, "Degradation of Poly(Methyl Methacrylate) in Solution," I\&EC Research, 35, 1795 (1996a).

Madras, G., J.M. Smith, B.J. McCoy, "Thermal Degradation of Poly( $\alpha$-Methylstyrene) in Solution," Poly. Deg. and Stab., 52, 349 (1996b).

Madras, G., J.M. Smith, B.J. McCoy, "Thermal Degradation Kinetics of Polystyrene in Solution," Poly. Deg. and Stab., (1996c); In press.

Madras, G., G.Y. Chung, J.M. Smith, B.J. McCoy, "Molecular Weight Effect on the Dynamics Of Polystyrene Degradation," I\&EC Research, (1997); In press.

Madras, G. and McCoy, "Effect of Hydrogen Donors on Polymer Degradation," Energy and Fuels, (1997); In review.

McCaffrey, W.C., Brues, M.J.; Cooper, D.G.; Kamal, M.R., "Thermolysis of Polyethylene Polystyrene Mixtures," J. App. Poly. Sci., 60, 2133 (1996).

Miller, A. "Industry Invests in Reusing Plastics," Env. Sci. Tech., 28, 16A (1994).

Murakata, T.; Saito, Y.; Yosikawa, T.; Suzuki, T.; Sato, S. "Solvent Effect on Thermal Degradation of Polystrene and Poly- $\alpha$-methylstyrene," Polymer, 34, 1436 (1993).

Nigam, A; Fake, D. M; Klein M.T. "Simple Approximate Rate Law for Both Short-Chain and Long Chain Rice Herzfeld Kinetics," AIChE J., 40, 908 (1994).

Ng, S.H, Seoud, H., Stanciulescu, M., Sugimoto, Y. "Conversion of Polyethylene to 
Transportation Fuels through Pyrolysis and Catalytic Cracking," Energy and Fuels, 9, 735 (1995).

Roy, M.; Rollin, A.L.; Schreiber, H.P. "Value Recovery from Polymer Wastes by

Pyrolysis," Poly. Eng. Sci., 18, 721 (1978).

Sato, S.; Murakata, T.; Baba, S.; Saito, Y.; Watanabe, S. "Solvent Effect on Thermal Degradation of Polystyrene," J. Appl. Poly. Sci., 40, 2065 (1990).

Wang, M., J.M. Smith, B.J. McCoy, "Continuous Kinetics for Thermal Degradation of Polymer in Solution," AIChE J., 41, 1521 (1995).

Wu, C.H.; Chang, C. Y.; Hor, J.L.; Shih, S.M.; Chen, L.W.; Chang, F.W., "On the Thermal Treatment of Plastic Mixtures : Pyrolysis Kinetics," Waste Management, 13, 221, (1993). 


\section{Table 1. Distribution Kinetics of Primary Reactions.}

Stoichiometric kernels: $\Omega\left(\mathrm{x}, \mathrm{x}^{\prime}\right)=1 / \mathrm{x}^{\prime}, \Omega\left(\mathrm{x}_{\mathrm{S}}, \mathrm{x}^{\prime}\right)=\delta\left(\mathrm{x}-\mathrm{x}_{\mathrm{S}}\right), \Omega\left(\mathrm{x}-\mathrm{x}_{\mathrm{S}}, \mathrm{x}^{\prime}\right)=\delta\left[\mathrm{x}-\left(\mathrm{x}^{\prime}-\mathrm{x}_{\mathrm{S}}\right)\right]$

\begin{tabular}{|c|c|c|c|}
\hline $\begin{array}{l}\text { Reaction } \\
\text { Type }\end{array}$ & Primary Reaction & Rate Expressions & $\begin{array}{l}\text { Moment Expressions } \\
(\mathrm{k} \text { is independent of } \mathrm{MW})\end{array}$ \\
\hline $\begin{array}{l}\text { Trans- } \\
\text { formation }\end{array}$ & $\mathrm{R}(\mathrm{x}) \rightarrow \mathrm{P}(\mathrm{x})$ & $\partial \mathrm{p} / \partial \mathrm{t}=-\partial \mathrm{r} / \partial \mathrm{t}=\mathrm{kr}(\mathrm{x}, \mathrm{t})$ & $\mathrm{dp}^{(\mathrm{n}) / \mathrm{dt}}=-\mathrm{dr} \mathrm{r}^{(\mathrm{n})} / \mathrm{dt}=\mathrm{kr}^{(\mathrm{n})}(\mathrm{t})$ \\
\hline $\begin{array}{l}\text { Random- } \\
\text { chain } \\
\text { scission }\end{array}$ & $\begin{array}{r}\mathrm{P}\left(\mathrm{x}^{\prime}\right) \rightarrow \mathrm{Q}(\mathrm{x})+ \\
\mathrm{R}\left(\mathrm{x}^{\prime}-\mathrm{x}\right)\end{array}$ & $\begin{array}{l}\partial \mathrm{p} / \partial \mathrm{t}=-\mathrm{k} \mathrm{p}(\mathrm{x}, \mathrm{t}) \\
\partial \mathrm{q} / \partial \mathrm{t}=\mathrm{k} \int_{\mathrm{x}}^{\infty} \mathrm{p}\left(\mathrm{x}^{\prime}, \mathrm{t}\right) \Omega\left(\mathrm{x}, \mathrm{x}^{\prime}\right) \mathrm{dx^{ \prime }} \\
=\partial \mathrm{r} / \partial \mathrm{t}\end{array}$ & 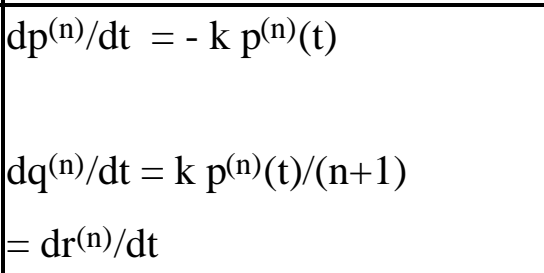 \\
\hline $\begin{array}{l}\text { Chain-end } \\
\text { scission }\end{array}$ & $\mid \begin{array}{l}\mathrm{P}\left(\mathrm{x}^{\prime}\right) \rightarrow \mathrm{Q}\left(\mathrm{x}_{\mathrm{S}}\right)+ \\
\mathrm{R}\left(\mathrm{x}^{\prime}-\mathrm{x}_{\mathrm{S}}\right) \\
\mathrm{x}_{\mathrm{S}} \text { is the } \mathrm{MW} \text { of th } \\
\text { specific product }\end{array}$ & $\begin{array}{l}\partial \mathrm{p} / \partial \mathrm{t}=-\mathrm{kp}\left(\mathrm{x}^{\prime}, \mathrm{t}\right) \\
\partial \mathrm{q} / \partial \mathrm{t}=\mathrm{k} \int_{\mathrm{x}}^{\infty} \mathrm{p}\left(\mathrm{x}^{\prime}, \mathrm{t}\right) \Omega\left(\mathrm{x}_{\mathrm{S}}, \mathrm{x}^{\prime}\right) \mathrm{dx} \\
\partial \mathrm{r} / \partial \mathrm{t}=\mathrm{k} \int_{\mathrm{x}}^{\infty} \mathrm{p}\left(\mathrm{x}^{\prime}, \mathrm{t}\right) \Omega\left(\mathrm{x}-\mathrm{x}_{\mathrm{S}}, \mathrm{x}^{\prime}\right) \\
\mathrm{dx^{ \prime }}\end{array}$ & $\begin{array}{l}\mathrm{dp}^{(\mathrm{n}) / \mathrm{dt}}=-\mathrm{k} \mathrm{p} \mathrm{p}^{(\mathrm{n})}(\mathrm{t}) \\
\mathrm{dq}^{(\mathrm{n}) / \mathrm{dt}}=-\mathrm{k} \mathrm{x}_{\mathrm{S}} \mathrm{n}^{\mathrm{q}^{(0)}(\mathrm{t})} \\
\mathrm{dr}^{(\mathrm{n}) / \mathrm{dt}}=\mathrm{k} \sum_{j=0}^{\mathrm{n}}\left({ }_{\mathrm{j}}^{\mathrm{n}}\right) \mathrm{x}_{\mathrm{s}}^{\mathrm{j}}(-1)^{\mathrm{j}} \mathrm{p}^{(\mathrm{n}-\mathrm{j})}\end{array}$ \\
\hline $\begin{array}{l}\text { Addition } \\
\text { reaction }\end{array}$ & $\begin{array}{l}P\left(x^{\prime}\right)+Q\left(x-x^{\prime}\right) \\
\rightarrow \quad R(x)\end{array}$ & $\begin{array}{l}\partial \mathrm{p} / \partial \mathrm{t}=-\mathrm{k} \mathrm{p}(\mathrm{x}, \mathrm{t}) \int_{0}^{\infty} \mathrm{q}\left(\mathrm{x}^{\prime}, \mathrm{t}\right) \mathrm{dx} \mathrm{x}^{\prime} \\
\partial \mathrm{q} / \partial \mathrm{t}=-\mathrm{k} \mathrm{q}(\mathrm{x}, \mathrm{t}) \int_{0}^{\infty} \mathrm{p}\left(\mathrm{x}^{\prime}, \mathrm{t}\right) \mathrm{dx^{ \prime }} \\
\partial \mathrm{r} / \partial \mathrm{t}=\mathrm{k} \int_{0}^{\mathrm{x}} \mathrm{p}\left(\mathrm{x}^{\prime}, \mathrm{t}\right) \mathrm{q}\left(\mathrm{x}-\mathrm{x}^{\prime}, \mathrm{t}\right) \\
\mathrm{dx^{ \prime }}\end{array}$ & $\begin{array}{l}\mathrm{d} \mathrm{p}^{(\mathrm{n})} / \mathrm{dt}=-\mathrm{k} \mathrm{p}^{(\mathrm{n})}(\mathrm{t}) \mathrm{q}^{(0)}(\mathrm{t}) \\
\mathrm{dq} \mathrm{q}^{(\mathrm{n}) / \mathrm{dt}}=-\mathrm{k} \mathrm{q}^{(\mathrm{n})}(\mathrm{t}) \mathrm{p}^{(0)}(\mathrm{t}) \\
\mathrm{dr} \mathrm{r}^{(\mathrm{n}) / \mathrm{dt}}=\mathrm{k} \sum_{j=0}^{\mathrm{n}}\left(\mathrm{n}_{j}\right) \mathrm{p}^{(\mathrm{j})}(\mathrm{t}) \mathrm{q}^{(\mathrm{n}-\mathrm{j})}\end{array}$ \\
\hline
\end{tabular}




\section{Figure captions}

1. Calibration curve of retention time, $t_{r}$, versus $\log M W\left(\log M W=13.58-0.68 t_{r}+\right.$ $\left.9.68 \times 10^{-3} \mathrm{t}_{\mathrm{r}}^{2}\right)$.

2. MWD of the inert solvent, mineral oil.

3. Plot of $\ln \left(\mathrm{p}_{\mathrm{A}}{ }^{(0)} / \mathrm{p}_{\mathrm{A} 0}{ }^{(0)}\right)$ versus time for polystyrene degradation at $275^{\circ} \mathrm{C}$ for four PAMS concentrations.

Polystyrene (2/L) only

Polystyrene $(2 \mathrm{~g} / \mathrm{L})+$ PAMS $(2 \mathrm{~g} / \mathrm{L})$.

Polystyrene (2 $\mathrm{g} / \mathrm{L})+$ PAMS $(5 \mathrm{~g} / \mathrm{L})$.

Polystyrene (2 g/L) + PAMS (10 g/L).

4. Effect of PAMS mass concentration, $\mathrm{p}_{\mathrm{B} 0}{ }^{(1)}$, on the rate coefficient of random chain scission, $\mathrm{k}_{\mathrm{r}}$, of polystyrene at $275^{\circ} \mathrm{C}$ plotted as $\mathrm{k}_{\mathrm{r}}$ versus $1 / \mathrm{p}_{\mathrm{B} 0}{ }^{(1)}$, as given by equation 1.45. The inset shows $\mathrm{k}_{\mathrm{r}}$ versus $\mathrm{p}_{\mathrm{B} 0}{ }^{(1)}$. 


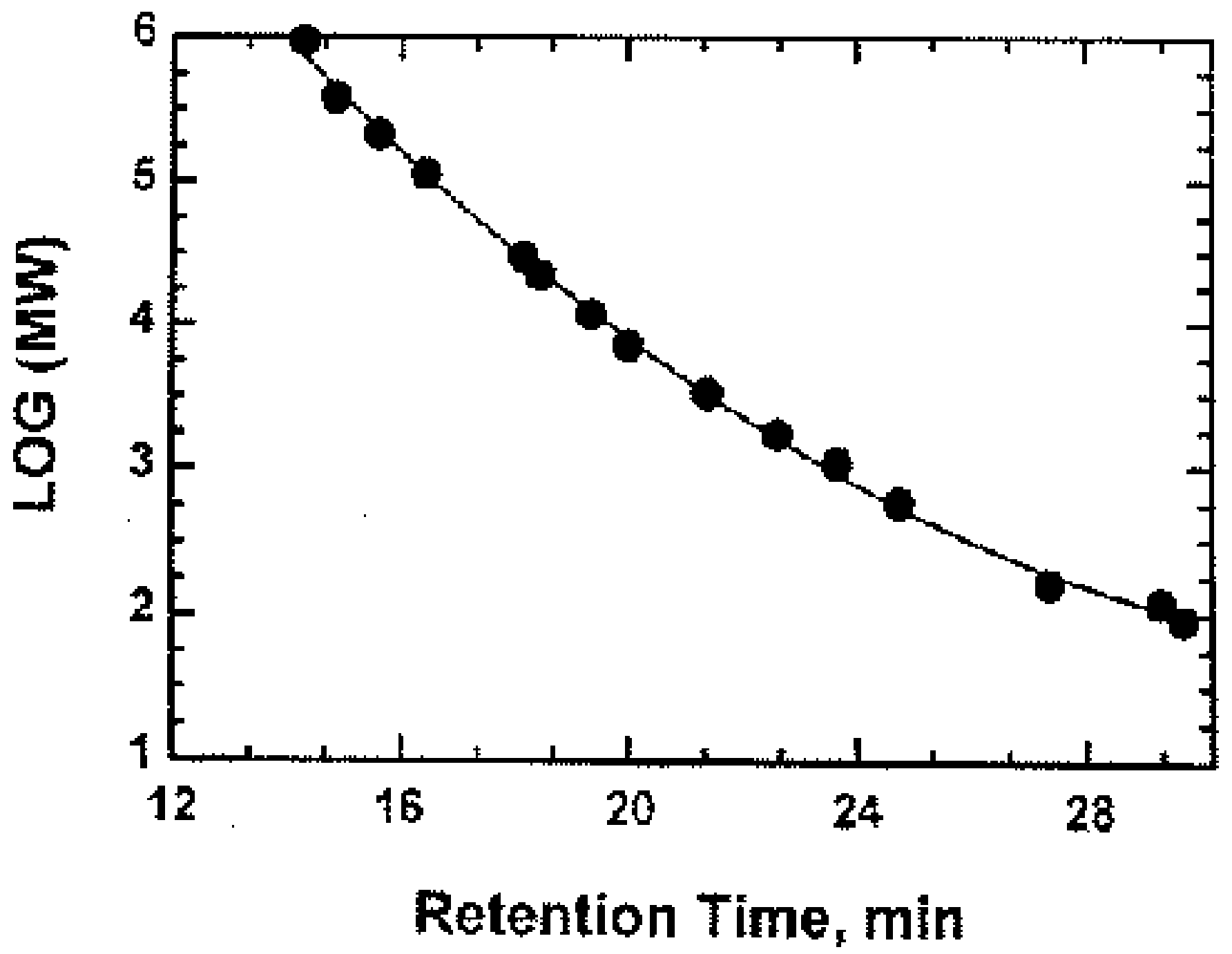

Figure 1. 


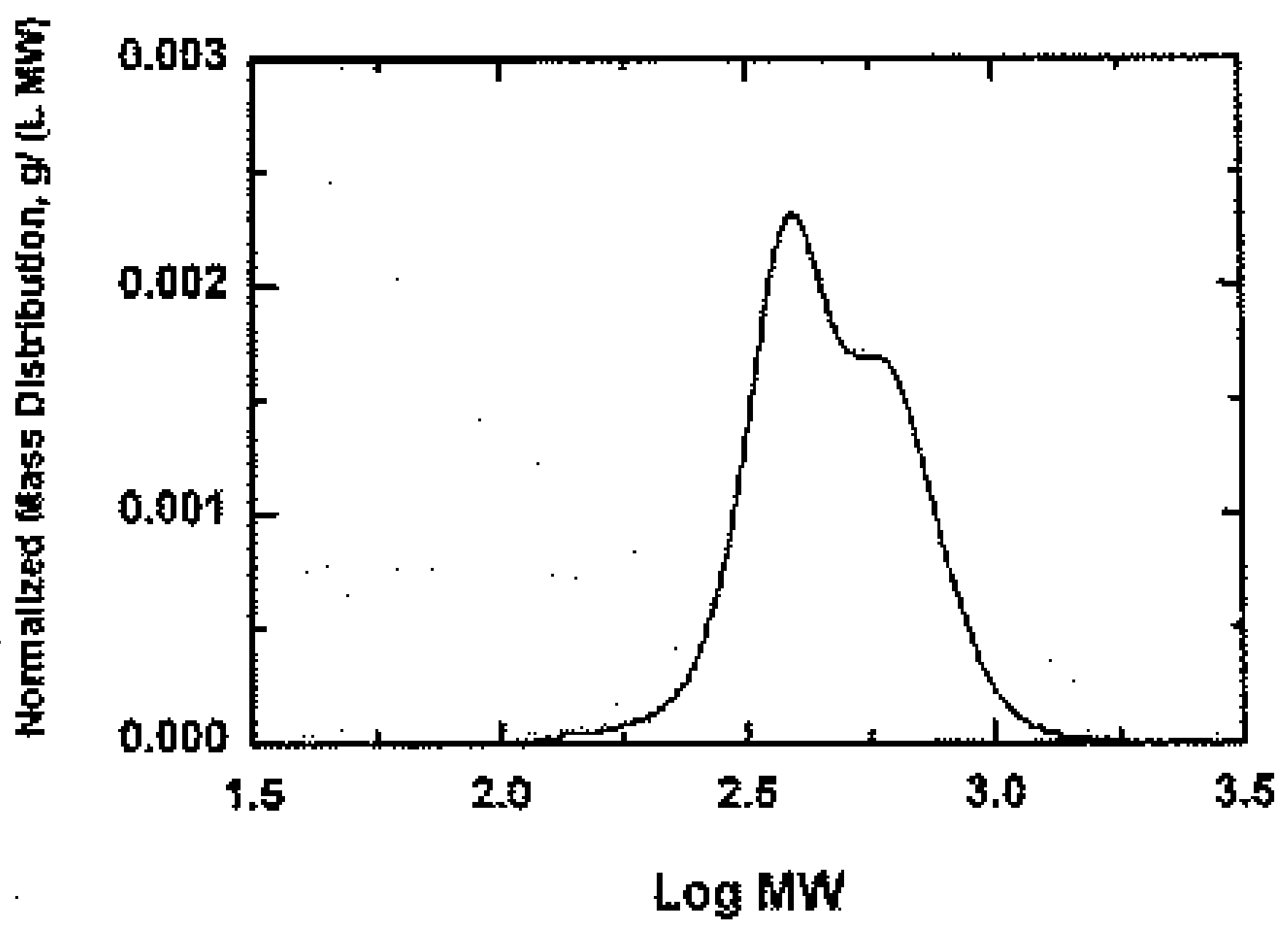

Figure 2. 


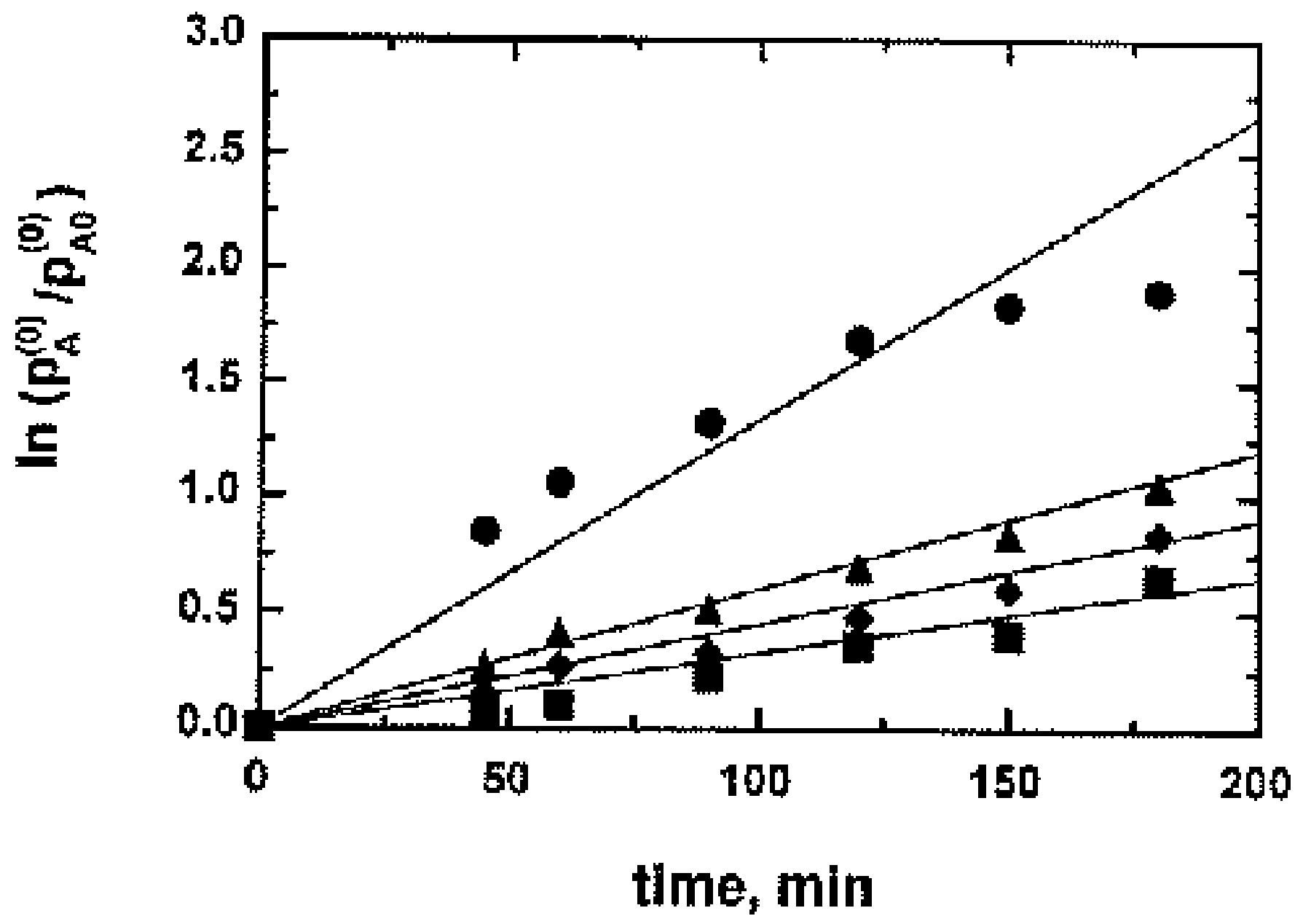

Figure 3. 


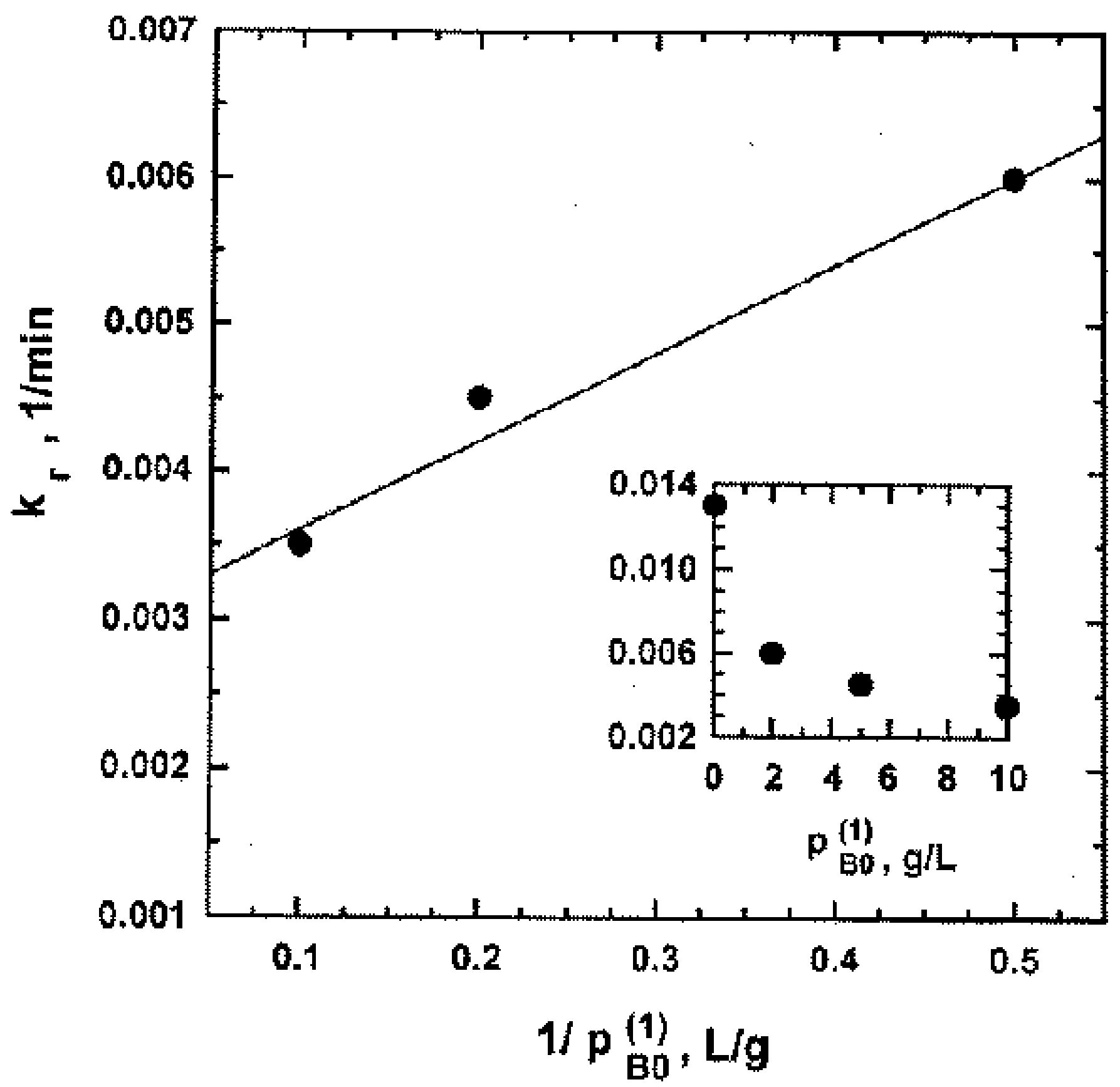

Figure 4. 